\title{
ASSESSMENT OF THE EFFECT OF STRONTIUM, LEAD AND ALUMINUM ON DUAL-ENERGY X-RAY ABSORPTIOMETRY AND QUANTITATIVE ULTRASOUND USING TRABECULAR BONE-MIMICKING PHANTOMS
}

\author{
by \\ Deok Hyun (Danny) Jang \\ Bachelor of Science in Life Science and X-ray Technology, \\ Queen’s University, Kingston, Ontario, Canada, 2011 \\ Bachelor of Science in Health Physics and Radiation Science, \\ University of Ontario Institute of Technology (UOIT), Oshawa, Ontario, Canada, 2014 \\ A thesis presented to Ryerson University \\ in partial fulfillment of the \\ requirements for the degree of \\ Master of Science \\ in the program of \\ Biomedical Physics
}

Toronto, Ontario, Canada, 2016

(C) Deok Hyun (Danny) Jang, 2016 


\section{AUTHOR'S DECLARATION}

I hereby declare that I am the sole author of this thesis. This is a true copy of the thesis, including any required final revisions, as accepted by my examiners.

I authorize Ryerson University to lend this thesis to other institutions or individuals for the purpose of scholarly research.

I further authorize Ryerson University to reproduce this thesis by photocopying or by other means, in total or in part, at the request of other institutions or individuals for the purpose of scholarly research.

I understand that my thesis may be made electronically available to the public.

Deok Hyun Jang 


\title{
ABSTRACT
}

\author{
ASSESSMENT OF THE EFFECT OF STRONTIUM, LEAD AND ALUMINUM ON \\ DUAL-ENERGY X-RAY ABSORPTIOMETRY AND QUANTITATIVE ULTRASOUND \\ MEASUREMENTS USING TRABECULAR BONE-MIMICKING PHANTOMS
}

\author{
Deok Hyun Jang \\ Master of Science, 2016 \\ Biomedical Physics \\ Department of Physics, Ryerson University
}

Dual-energy X-ray absorptiometry (DXA) is the gold standard of bone densitometry. However, inaccurate estimation of areal bone mineral density (aBMD) is of concern when calcium in bone mineral is partially substituted with bone-seeking elements such as strontium, lead and aluminum. Quantitative ultrasound (QUS) is an alternative bone densitometry technique that can assess bone health based on the measurement of acoustic parameters. This study aims to investigate the effect of the clinically relevant concentrations of bone-seeking elements on aBMD measured by DXA and the acoustic parameters measured by QUS, using trabecular bonemimicking phantoms. Statistically significant linear relationship was observed between aBMD and strontium concentration. For clinically relevant concentrations of lead and aluminum, the deviation in aBMD measurements was within $1 \%$ coefficient of variation of DXA. No statistically significant deviation was observed in stiffness index measurement by QUS in the presence of any of the three bone-seeking elements. 


\section{ACKNOWLEDEMENTS}

I would like to thank my supervisors Dr. Ana Pejović-Milić and Dr. Jahan Tavakkoli for their expert advices and guidance throughout my study. Your continuous moral support and encouragement really meant a lot to me, and were crucial for the completion of this work.

I would like to thank Bisma Rizvi for guiding me through the essential techniques of phantom preparation and operation of the QUS device.

I would like to thank Eric Da Silva for his expertise in chemistry, which was needed for the phantom preparation, his role as a member of the supervisory committee and assistance in the analysis of the acquired data.

I would like to thank Arthur Worthington, Luke Yaraskavitch and Graham Ferrier for their technical assistance.

I would like to thank Dr. Luba Slatkovska and Dr. Angela M. Cheung for their expertise in clinical bone densitometry.

I would like to thank Queenie Wong and Diana Yau for the operation of DXA unit.

I would like to thank my supervisory committee member Dr. Raffi Karshafian, defence committee member Dr. James Gräfe, program director Dr. Carl Kumaradas, administrative assistant Tess Sy, and program administrators Adriana Gaertner and Sophia Finos.

Finally, I would like to thank my family for their unconditional support.

This work is supported by NSERC Discovery grants that were awarded to Dr. Ana Pejović -Milić and Dr. Jahan Tavakkoli. 


\section{TABLE OF CONTENTS}

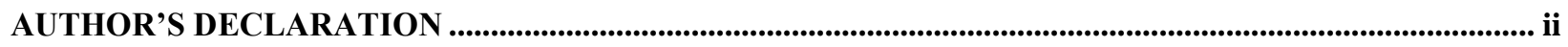

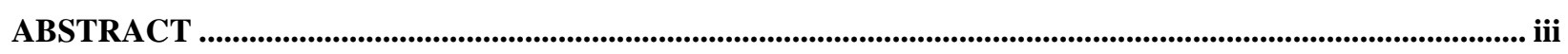

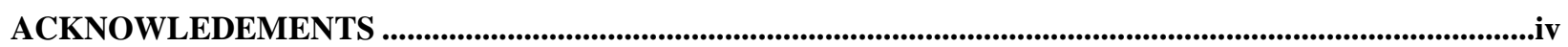

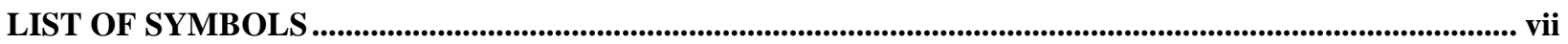

LIST OF ABBREVIATIONS ..............................................................................................................................

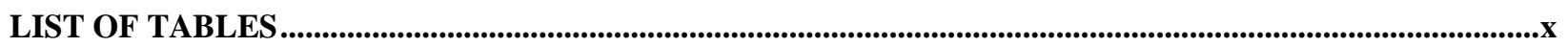

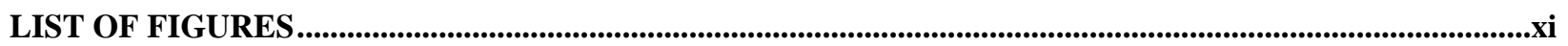

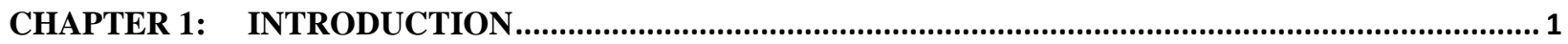

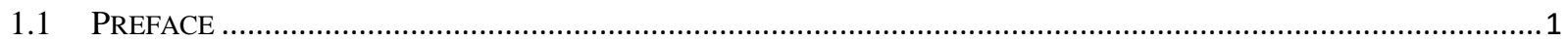

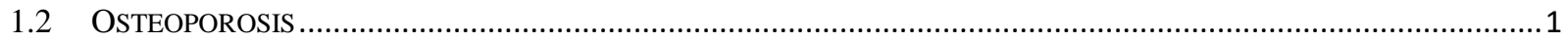

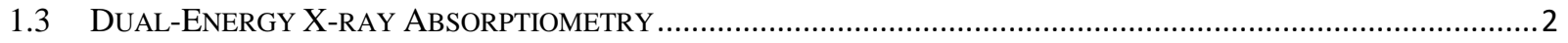

1.3.1 Physics of X-ray Photon Attenuation ...........................................................................................

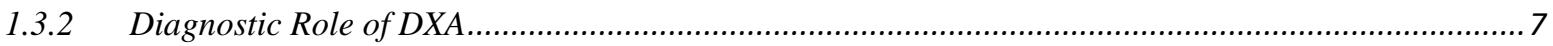

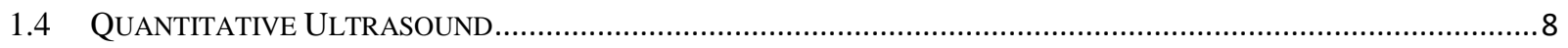

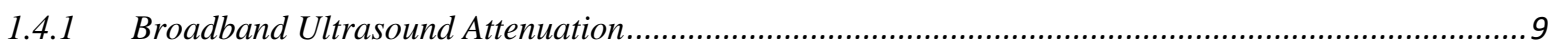

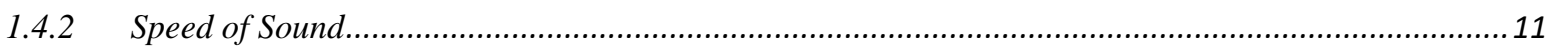

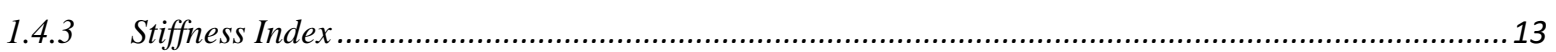

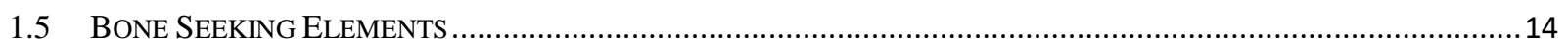

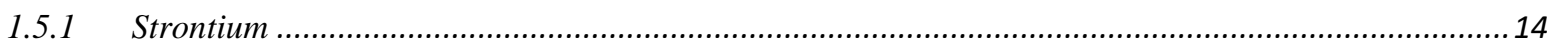

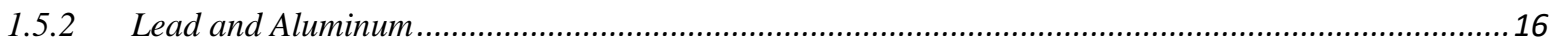

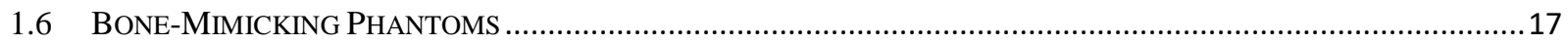

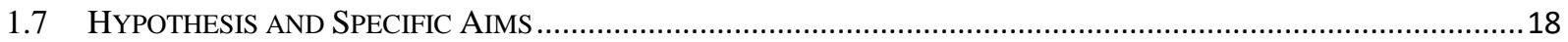

CHAPTER 2: ASSESSMENT OF THE EFFECT OF STRONTIUM, LEAD AND ALUMINUM ON

DUAL-ENERGY X-RAY ABSORPTIOMETRY AND QUANTITATIVE ULTRASOUND

MEASUREMENTS USING TRABECULAR BONE-MIMICKING PHANTOMS........................................ 20

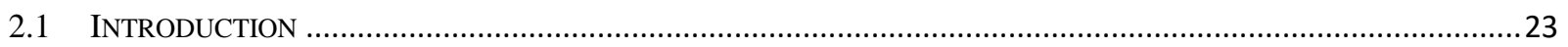

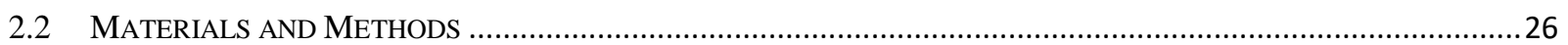

2.2.1 Hydroxyapatite-based trabecular bone-mimicking phantom...........................................................26

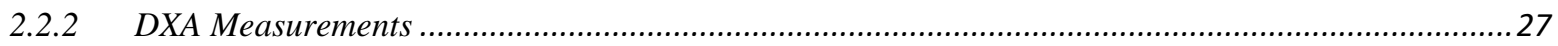

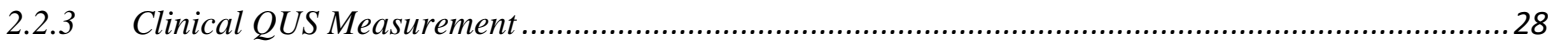

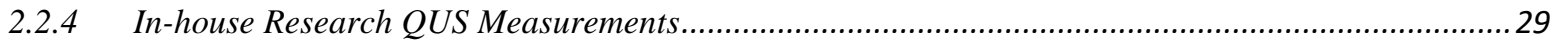

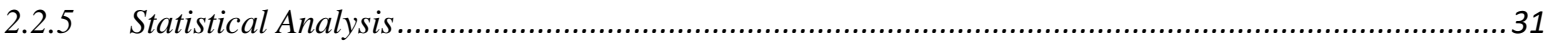




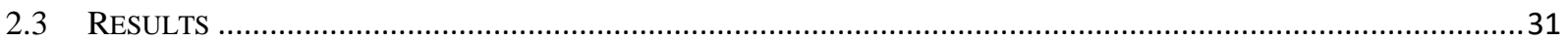

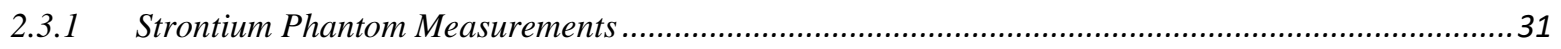

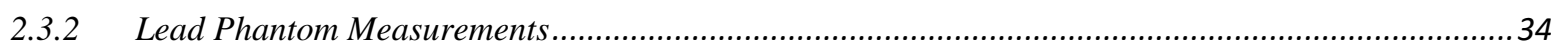

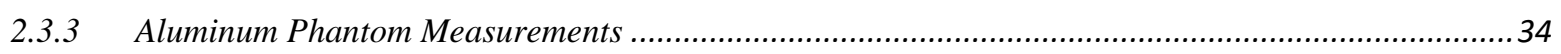

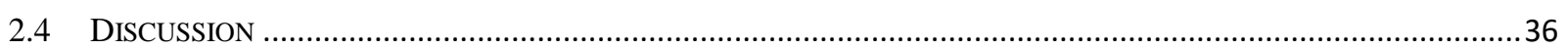

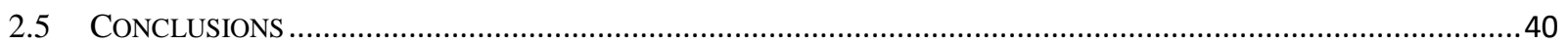

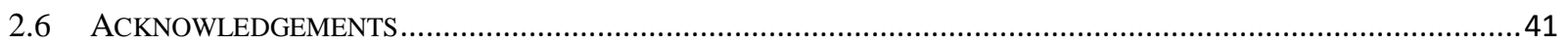

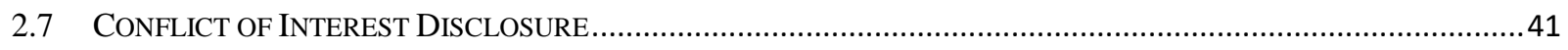

CHAPTER 3: SUMMARY, CONCLUSIONS AND FUTURE WORK .............................................. 42

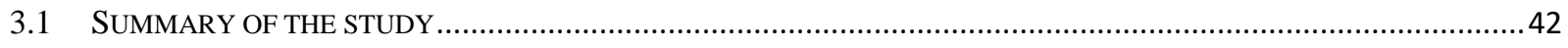

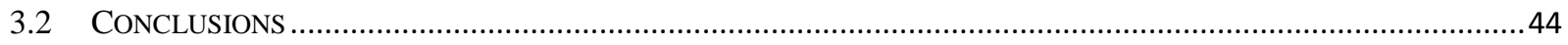

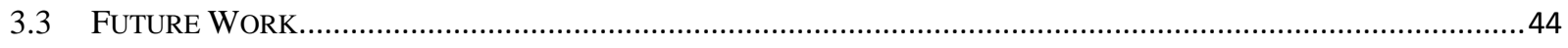

APPENDIX A: PROCEDURE FOR PRODUCING TRABECULAR BONE-MIMICKING PHANTOMS....... 46

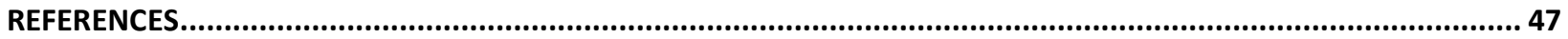




\section{LIST OF SYMBOLS}

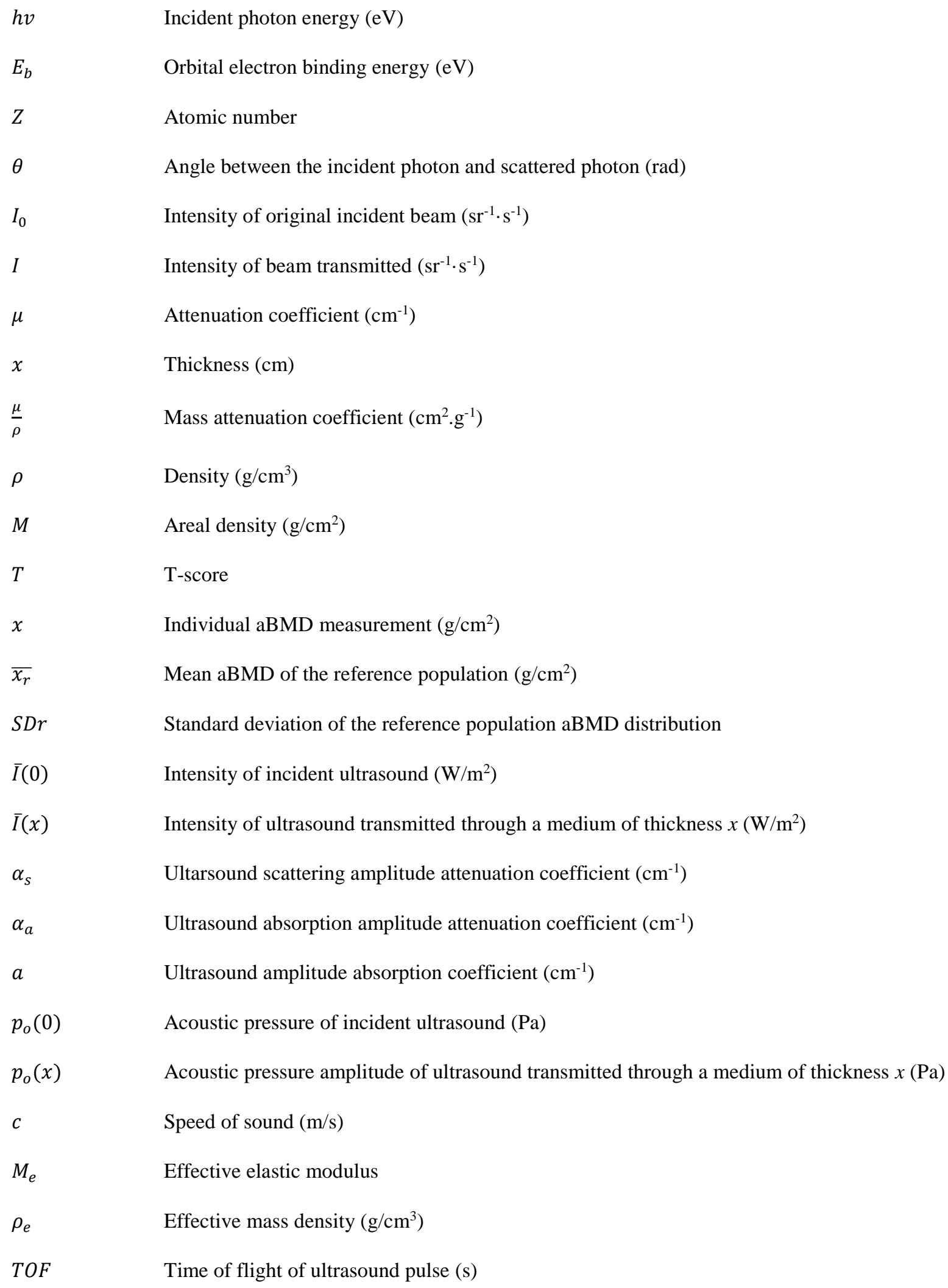

$M_{e} \quad$ Effective elastic modulus

Orbital electron binding energy $(\mathrm{eV})$

Atomic number

Angle between the incident photon and scattered photon (rad)

Intensity of original incident beam $\left(\mathrm{sr}^{-1} \cdot \mathrm{s}^{-1}\right)$

Intensity of beam transmitted $\left(\mathrm{sr}^{-1} \cdot \mathrm{s}^{-1}\right)$

Attenuation coefficient $\left(\mathrm{cm}^{-1}\right)$

Thickness (cm)

Mass attenuation coefficient $\left(\mathrm{cm}^{2} \cdot \mathrm{g}^{-1}\right)$

Density $\left(\mathrm{g} / \mathrm{cm}^{3}\right)$

Areal density $\left(\mathrm{g} / \mathrm{cm}^{2}\right)$

T-score

Individual aBMD measurement $\left(\mathrm{g} / \mathrm{cm}^{2}\right)$

Mean aBMD of the reference population $\left(\mathrm{g} / \mathrm{cm}^{2}\right)$

Standard deviation of the reference population aBMD distribution

Intensity of incident ultrasound $\left(\mathrm{W} / \mathrm{m}^{2}\right)$

Intensity of ultrasound transmitted through a medium of thickness $x\left(\mathrm{~W} / \mathrm{m}^{2}\right)$

Ultarsound scattering amplitude attenuation coefficient $\left(\mathrm{cm}^{-1}\right)$

Ultrasound absorption amplitude attenuation coefficient $\left(\mathrm{cm}^{-1}\right)$

Ultrasound amplitude absorption coefficient $\left(\mathrm{cm}^{-1}\right)$

Acoustic pressure of incident ultrasound $(\mathrm{Pa})$

Acoustic pressure amplitude of ultrasound transmitted through a medium of thickness $x(\mathrm{~Pa})$

Speed of sound $(\mathrm{m} / \mathrm{s})$

Time of flight of ultrasound pulse (s) 
TOF ${ }^{r e f}$

C

$S r \%$

Time of flight of ultrasound pulse through the reference medium (s)

Strontium adjustment factor

Strontium ratio $\mathrm{Sr} /(\mathrm{Sr}+\mathrm{Ca})(\%)$ 


\section{LIST OF ABBREVIATIONS}

$\begin{array}{ll}\text { aBMD } & \text { Areal bone mineral density } \\ \text { BMC } & \text { Bone mineral content } \\ \text { BUA } & \text { Broadband ultrasound attenuation } \\ \text { DPA } & \text { Dual Photon Absorptiometry } \\ \text { DXA } & \text { Dual-energy x-ray absorptiometry } \\ \text { QUI } & \text { Quantitative ultrasound index } \\ \text { QUS } & \text { Quantitative ultrasound } \\ \text { SOS } & \text { Speed of sound } \\ \text { SI } & \text { Stiffness Index } \\ \text { vBMD } & \text { Volumetric bone mineral density }\end{array}$




\section{LIST OF TABLES}

Table 2.1. The linear regression analysis for the measurements done on the strontium multilayer

trabecular bone phantoms

Table 2.2. The linear regression analysis for the measurements done on the lead multilayer trabecular bone phantoms.

Table 2.3. The linear regression analysis for the measurements done on the aluminium multilayer trabecular bone phantoms. 


\section{LIST OF FIGURES}

Fig. 1.1. Principles of ultrasound transverse transmission at the calcaneus to measure BUA. (a) Placement of transducers in the mediolateral direction, (b) substitution method of assessing BUA, (c) difference in the amplitude signal obtained from the reference medium (solid line) and through the heel (dashed line), (d) difference in the amplitude spectra of the reference signal (solid line) and of the signal transmitted through the heel (dashed line) (e) frequency dependent

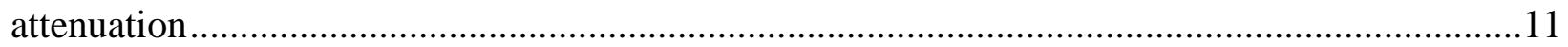

Fig. 2.1. Schematic experimental setup of the DXA measurements. ........................................28

Fig. 2.2. Schematic design of the multi-layer trabecular bone-mimicking phantom that mimics

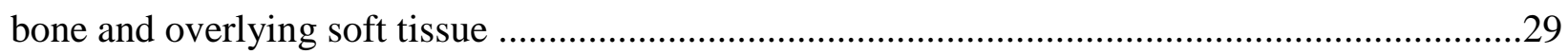

Fig. 2.3. Schematic experimental set up of the in-house researech QUS system........................30

Fig. 2.4. BMD analysis result of the reference phantom by Hologic Horizon ${ }^{\circledR}$ DXA device........32

Fig. 2.5. Parameters measured by the DXA system and the two QUS system as a function of strontium concentration: (a) aBMD measured by DXA, (b) BUA measured by QUS, (c) SOS

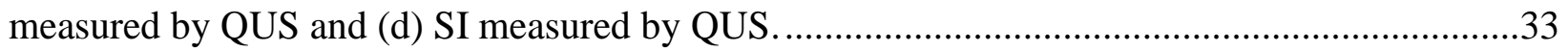

Fig. 2.6. Overestimation of aBMD as a function of strontium content. .....................................33

Fig. 2.7. Parameters measured by the DXA system and the two QUS system as a function of lead concentration: (a) aBMD measured by DXA, (b) BUA measured by QUS, (c) SOS measured by QUS and (d) SI measured by QUS. .35

Fig. 2.8. Parameters measured by the DXA system and the two QUS system as a function of aluminium concentration: (a) aBMD measured by DXA, (b) BUA measured by QUS, (c) SOS measured by QUS and (d) SI measured by QUS .36 


\section{CHAPTER 1:INTRODUCTION}

\subsection{Preface}

Chapter 2 of this thesis represents a manuscript titled "Assessment of the Effect of Strontium, lead and Aluminum on Dual-Energy X-Ray Absorptiometry and Quantitative Ultrasound Measurements Using Trabecular Bone-Mimicking Phantoms." The co-authors of the manuscript were Deok Hyun Jang, Eric Da Silva, Jahan Tavakkoli, Lubomira Slatkovska, Angela M. Cheung and Ana Pejović-Milić. The contributions of each co-author are as following:

Study Design: DJ, EDS, LS, JT, AMC and APM. Data Collection and Analysis: DJ. Data Interpretation: DJ, EDS, JT, AMC and APM. Drafting the Manuscript: DJ. Revising and Approving the Final Version of the Manuscript: DJ, EDS, LS, JT, AMC and APM.

\subsection{Osteoporosis}

Osteoporosis is a bone metabolic disorder characterized by the loss of bone mass and degeneration of bone microarchitecture. ${ }^{(1)}$ It is caused by bone remodeling imbalance due to shift of the equilibrium between bone reformation and reabsorption toward the later. ${ }^{(2)}$ Consequently, osteoporosis increases the risk of fragility fractures that occur with little or no apparent trauma, especially at sites such as hip, spine and wrists. ${ }^{(3)}$ Osteoporosis has considerable public health implication as fractures associated with osteoporosis has significant negative impact on quality of life, and increases morbidity and mortality. ${ }^{(4)}$

Osteoporosis is most prevalent among, but not limited to, post-menopausal women. In Canada, it is estimated that approximately 1 in 4 women and 1 in 8 men have osteoporosis. ${ }^{(5)}$ It is 
a major public health concern in Canada and worldwide, as the prevalence of osteoporosis is expected to rise steeply over next few decades due to the increase in overall life expectancy. ${ }^{(5)}$ Osteoporotic fractures incur heavy financial burden on Canadian healthcare system due to costs associated with to hospitalizations, emergency care, surgeries, rehabilitation, drugs, and productivity losses. ${ }^{(6)}$ It is estimated that the economic burden of osteoporosis related fractures in Canada was $\$ 1.3$ billion in 1993 and $\$ 2.3$ billion in 2010.(7) Therefore, the diagnosis of osteoporosis prior to the occurrence of fracture is imperative.

\subsection{Dual-Energy X-ray Absorptiometry}

Dual-Energy X-Ray Absorptiometry (DXA) is a diagnostic technique that uses two X-ray beams with different energies to measure areal bone mineral density (aBMD). DXA scans are most commonly performed at the lumbar spine and the proximal femur (hip), bones that are weight-bearing. In case a patient condition does not permit the examination of the two sites, the radius of the forearm can be assessed instead. DXA is the current gold standard of bone densitometry that is used to diagnose osteoporosis and evaluate the risk of fracture. The advantages of DXA include good precision, short scan times, low radiation dose, and effective quality control and calibration. ${ }^{(8)}$

X-ray beams are produced by accelerating free electrons from a heated filament toward an anode by applying electric potential. The collision of the accelerated electrons with the anode target generates poly-energetic bremsstrahlung radiation and characteristic X-ray, and the voltage across the cathode and anode determines the maximum energy of the X-ray. Two approaches were adapted by DXA manufacturers to differentiate the energies of the X-ray beams: K-absorption edge filtering (e.g. in the GE Lunar Prodigy ${ }^{\circledR}$ device) and $\mathrm{kVp}$ switching (e.g. in the Hologic 
Horizon ${ }^{\circledR}$ device). ${ }^{(9)}$ The K-absorption filtering method uses a metal filter to split one polyenergetic X-ray beam into high-energy and low-energy components. The kVp switching method produces two X-ray beams with different energy spectrum by switching the tube voltage continuously. Consequentially, the effective energy of the two beams vary among the manufacturers and models.

\subsubsection{Physics of X-ray Photon Attenuation}

The attenuation of the X-ray beams in diagnostic energy range can be attributed to photoelectric absorption and Compton scattering. ${ }^{(8)}$ Photoelectric absorption is the interaction between an incident photon and a tightly bound orbital electron of an attenuating medium. In this process, the incident photon is completely absorbed by the target electron and deposits all its energy to the electron. As the result the electron is ejected from the orbital of the target atom, and it is referred to as a photoelectron. The kinetic energy $E_{k}$ of the photoelectron can be obtained using the following equation:

$$
E_{k}=h v-E_{b}
$$

where $h v$ is the incident photon energy and $E_{b}$ is the binding energy of the orbital electron. The vacancy in the orbital created by the ejection of the electron is filled by a higher shell electron. During the process, a secondary characteristic X-ray or an Auger electron can be produced. The photoelectric attenuation coefficient is highly dependent on the atomic number $Z$ of an attenuating medium. The photoelectric attenuation coefficient is proportional to $Z^{4}$ for high $Z$ absorbers and $Z^{5}$ for low $Z$ absorbers. Conversely, in relation to increasing photon energy the photoelectric attenuation coefficient decreases as $1 /(h v)^{3}$. Therefore the photoelectric attenuation coefficient is approximately proportional to $Z^{4} /(h v)^{3}$. $^{(10)}$ 
Compton scattering is the interaction of an incident photon with a free orbital electron, whose binding energy $E_{b}$ is significantly smaller than the photon energy $h v$. Upon the collision of the photon with the free electron, the photon transfers part of its energy to the electron, allowing it to scatter, and the photon is scattered with diminished energy. The energy of the scattered photon $h v^{\prime}$ can be calculated from the equation:

$$
h v^{\prime}=\frac{h v}{1+\frac{h v}{m_{e} c^{2}}(1-\cos \theta)}
$$

where $h v$ is the incident photon energy, $m_{e} c^{2}$ is the electron rest energy $(0.511 \mathrm{MeV})$, and $\theta$ is the angle between the incident photon and scattered photon. The scattering photon has highest energy when $\theta=0$, and the lowest energy when $\theta=\pi$ (backscatter). Higher initial photon energy increase the probability of the forward scatter. The Compton atomic attenuation coefficient is proportional to the electron density which in turn is dependent on $Z$. However it is important to note that the mass attenuation coefficient for Compton scattering does not depend on $Z$ due to the normalization of the density. ${ }^{(10)}$ The Compton atomic attenuation coefficient decreases with increasing energy.

The total attenuation coefficient is the sum of attenuation coefficients for all photon interaction with a medium. Rayleigh scattering also known as coherent scattering, and pair production are other modes of photon interaction with a matter. However, the two modes of photon interactions hold little or no significance for DXA. The attenuation coefficient for Rayleigh scattering, which does not transfer any energy to a charged particle, is significantly lower than that of photoelectric absorption or Compton scattering. In addition, pair production requires photon energy above $2 m_{e} c^{2}(1.022 \mathrm{MeV})$ in order to produce an electron and a positron, and the photon energy relevant to DXA is below the threshold energy of photoelectric effect. Therefore, attenuation coefficient for photoelectric effect and Compton scattering determines the total 
attenuation coefficient. Since the attenuation coefficients of photoelectric absorption and Compton effect are $\mathrm{Z}$ dependent, bone with higher effective $Z$ than soft tissue consequentially have higher total attenuation coefficient. In addition, although the mass attenuation coefficient of Compton effect is $\mathrm{Z}$ independent, bone has higher mass attenuation coefficient than soft tissue mainly due to high $\mathrm{Z}$ dependency of photoelectric effect.

In practice, attenuation of photon can be evaluated using the ratio of the original intensity of an incident beam and the measured intensity of a beam transmitted through a medium. Attenuation of mono-energetic beam through a medium can be expressed using the following equation:

$$
I=I_{0} e^{-\mu x}
$$

where $I_{0}$ is the intensity of original incident beam, $I$ is the intensity of the beam transmitted, $x$ is the thickness of the medium (in $\mathrm{cm}$ ), and $\mu$ is the attenuation coefficient (in $\mathrm{cm}^{-1}$ ) specific to the medium and the energy of the beam. The above equation can be re-written using the mass attenuation coefficient, which takes the mass density of the medium into account.

$$
I=I_{0} e^{-\left(\frac{\mu}{\rho}\right) \rho x}
$$

In Equation 1.4, $\frac{\mu}{\rho}$ denotes mass attenuation coefficient of the medium (in $\mathrm{cm}^{2} / \mathrm{g}$ ), and $\rho$ denotes the mass density of the medium (in $\mathrm{g} / \mathrm{cm}^{3}$ ). The product of the mass density $\rho$ and the thickness $x$ yield the areal density denoted as $M$ (in $\mathrm{g} / \mathrm{cm}^{2}$ ). Assuming that the photon is passing through bone and soft tissue, Equation $1.4^{(9)}$ can be written as:

$$
\mathrm{I}=I_{0} e^{-\left\{\left[\left(\frac{\mu}{\rho}\right)_{\text {Bone }} \times M_{\text {Bone }}\right]+\left[\left(\frac{\mu}{\rho}\right)_{S T} \times M_{S T}\right]\right\}}
$$

Then, by normalizing $I$ with $I_{0}$ and taking the natural log of the ratio yields:

$$
-\ln \frac{I}{I_{0}}=\left[\left(\frac{\mu}{\rho}\right)_{\text {Bone }} \times M_{\text {Bone }}\right]+\left[\left(\frac{\mu}{\rho}\right)_{S T} \times M_{S T}\right]
$$


Since DXA uses two X-ray beams, another equation can be generated for a higher energy beam where prime is used to denote parameters for higher energy beam:

$$
-\ln \frac{I^{\prime}}{I_{0}^{\prime}}=\left[\left(\frac{\mu}{\rho}\right)_{\text {Bone }}^{\prime} \times M_{\text {Bone }}\right]+\left[\left(\frac{\mu}{\rho}\right)_{S T}^{\prime} \times M_{S T}\right]
$$

Based on the rearrangement of Equation 1.7 and 1.8, aBMD can be solved:

$$
M_{\text {Bone }}=\frac{\left[\left(\ln \frac{I^{\prime}}{I_{0}^{\prime}}\right)-\left(\frac{\left(\frac{\mu}{\rho}\right)^{\prime}}{\left(\frac{\mu}{\rho}\right)_{S T}}\right) \times\left(\ln \frac{I}{I_{0}}\right)\right]}{\left[\left(\frac{\mu}{\rho}\right)^{\prime}{ }_{\text {Bone }}-\left(\frac{\left(\frac{\mu}{\rho}\right)^{\prime}}{\left(\frac{\mu}{\rho}\right)_{S T}}\right) \times\left(\frac{\mu}{\rho}\right)_{\text {Bone }}\right]}
$$

The ratio of the mass attenuation coefficient of the soft tissue represented as $\frac{\left(\frac{\mu}{\rho}\right)^{\prime}}{\left(\frac{\mu}{\rho}\right)_{S T}}$, also known as $\mathrm{R}$-value, can be measured at a site where bone is not present in the beam pathway (i.e. $\left.M_{\text {Bone }}=0\right){ }^{(11)}$

$$
R=\frac{\left(\frac{\mu}{\rho}\right)_{S T}^{\prime}}{\left(\frac{\mu}{\rho}\right)_{S T}}=\left.\frac{\ln \frac{I^{\prime}}{I_{0}^{\prime}}}{\ln \frac{I}{I_{0}}}\right|_{M_{\text {Bone }}=0}
$$

It is important to note that the derivation of the above equations are simplified based on monochromatic beam approximation, more suited for Dual Photon Absorptiometry (DPA) that uses two mono-energetic beams originated from a radionuclide source. ${ }^{(9)}$ In reality, DXA emits two poly-energetic beams with different maximum energy. The poly-energetic nature of the beams is because the primary mode of X-ray production is via bremsstrahlung. Therefore, to take this into account, Equation 1.3 needs to be re-written using energy dependent intensity $I_{0}(E)$ as given by:

$$
I=\int I_{0}(E) e^{-\mu(E) x} d E
$$




\subsubsection{Diagnostic Role of DXA}

There is a systematic variation in measurement readings obtained from the same subject using DXA scanners from different manufacturers. This is because, among other things, DXA scanners from different manufacturers use different analysis algorithm, region of interest (ROI) definitions, calibration protocol and effective photon energies. ${ }^{(12)}$ Therefore, direct comparison among aBMD measurements obtained using different devices cannot be made. There has been efforts to standardize aBMD and BMC measurements among the manufactures, initiated by the International Committee for Standards in Bone Measurement (ICSBM), to remove the measurement difference among manufacturers using a correction formula. ${ }^{(13)}$ However, clinical application of standardization to compare results of an individual patients was not recommended due to the standard error associated with the correction. ${ }^{(14)}$

Therefore, the clinical diagnosis of osteoporosis largely relies on T-score and Z-score derived from aBMD. The T-score is the number of standard deviations above or below the mean for a young healthy reference population. ${ }^{(15)}$ Derivation of T-score can be represented using Equation 1.11 where $T$ denotes T-score, $x$ denotes an individual result, $\overline{x_{r}}$ denotes the mean of the reference population, and SDr denotes the standard deviation of the reference population distribution:

$$
T=\frac{x-\overline{x_{r}}}{S D r}
$$

Similarly, Z-score is the number of standard deviations above or below the mean for an individual's age, sex and ethnicity.

For the screening purpose, Osteoporosis Society of Canada recommends aBMD assessment to individuals over age of 65 , or to younger adults with identifiable risk factors that increases the susceptibility to bone loss. ${ }^{(16)}$ The risk factors include history of fragility fracture, hypogonadism, use of high-risk medications, hyperparathyroidism, high alcohol consumption and smoking. 
Traditionally the diagnosis of osteoporosis was based on the diagnostic criteria established by the World Health Organization (WHO), which defines osteoporosis as aBMD T-score below 2.5. (17) For individuals under age of 50, Z-score with a threshold of -2.0 is used to determine if aBMD result is within or below the expected range for age. More detailed fracture risk evaluation systems, which account for the other risk factors in addition to T-score, have been adapted by Osteoporosis Canada (OC) recently in order to better reflect the complex nature of the fracture risk $^{(16)}$ : Canadian Association of Radiologists and Osteoporosis Canada (CAROC) system and Fracture Risk Assessment Tool (FRAX) of the WHO calibrated for Canadian population.

\subsection{Quantitative Ultrasound}

Quantitative ultrasound (QUS) is a bone densitometry technique that assesses bone health by measuring ultrasonic parameters. The main advantages of QUS over DXA include its portability, relatively low cost and absence of exposure to ionizing radiation. ${ }^{(18)}$ QUS measurements are commonly taken at the calcaneus using transverse transmission technique to obtain broadband ultrasound attenuation (BUA) and speed of sound (SOS). The calcaneus is desired site of examination because it is a weight bearing bone composed mainly of trabecular bone, and has flat and parallel medial and lateral surface ideal for coupling of two transducers. ${ }^{(19)}$ The transverse transmission system consists of two ultrasound transducers placed on opposite sides, where one functions as a transmitter and another functions as a receiver. There are two designs of QUS available for the transverse transmission technique: a water-based system and a dry contact system. $^{(15)}$ The first clinical devices developed were water-based system. For this system, the heel is immersed in water and placed between two transducers. ${ }^{(15)}$ Dry contact design was later developed and it tend to replace water-based system; it has easier portability, better hygiene and 
higher precision as water temperature has significant impact on ultrasonic parameters. ${ }^{(15)}$ The two transducers in dry system are mechanically driven to maintain constant pressure in direct contact with the heel.

\subsubsection{Broadband Ultrasound Attenuation}

Similar to photon attenuation, ultrasound attenuation involves decrease in the intensity of ultrasound wave with respect to increasing the wave propagation distance. Absorption and scattering are the two main mechanisms that contribute to ultrasonic attenuation. ${ }^{(15)}$ During absorption process, ultrasound energy is converted into other forms of energy such as heat, chemical energy and light. Scattering of ultrasound represents redirection of ultrasound wave along paths that differ from the incident wave. Because scattering phenomena is the interaction between ultrasound wave and boundaries of heterogeneous particles, there would be no scattering for pure fluids for which attenuation and absorption become identical.

If the original ultrasound incident intensity is $\bar{I}(0)$, then intensity of the ultrasound wave after passing an attenuating medium with thickness $x$ can be represented as:

$$
\bar{I}(x)=\bar{I}(0) e^{-2\left(\alpha_{s}+\alpha_{a}\right) x}=\bar{I}(0) e^{-2 \alpha x}
$$

where $\alpha$ is amplitude attenuation, which is the sum of absorption amplitude attenuation coefficient, $\alpha_{a}$, and scattering amplitude attenuation coefficient, $\alpha_{s}$. The amplitude attenuation coefficient is frequency, temperature and pressure dependent. The unit of the attenuation coefficient is $\mathrm{cm}^{-1}$, but in practice Nepers per centimeter $(\mathrm{Np} / \mathrm{cm})$ or decibel per $\mathrm{cm}(\mathrm{dB} / \mathrm{cm})$ is used. The conversion between $\mathrm{Np} / \mathrm{cm}$ and $\mathrm{dB} / \mathrm{cm}$ is $\alpha_{d B}=8.686 \alpha_{N p}$ or $\alpha_{N p}=0.1151 \alpha_{d B} \cdot{ }^{(15)}$ The attenuation coefficient in $\mathrm{dB} / \mathrm{cm}$ can be written in terms of intensity or pressure ratios using following equations:

$$
\alpha_{d B}=\frac{10}{x} \log \left[\frac{\bar{I}_{o}(0)}{\bar{I}_{o}(x)}\right]
$$




$$
\alpha_{d B}=\frac{20}{x} \log \left[\frac{p_{o}(0)}{p_{o}(x)}\right]
$$

Attenuation is calculated as $\alpha_{d B} x$ using Equation 1.14, where $p_{o}(0)$ is the pressure measured without the calcaneus, and $p_{o}(x)$ is the pressure measured with the calcaneus with thickness of $x$ in place. A quasi-linear relationship between ultrasonic attenuation and frequency has been observed for bone between frequency range of 0.2 to $2 \mathrm{MHz} .{ }^{(19)}$ Based on this relationship, BUA (with units of $\mathrm{dB} / \mathrm{MHz}$ ) is determined by taking the slope of the linear regression of attenuation and frequency. BUA is often normalized by the thickness in order to evaluate thickness independent attenuation coefficient rather than total attenuation. However, BUA is used in clinical practice because normalized BUA measurement does not show significant improvement in fracture discrimination. $^{(20)}$ Typically, the clinical QUS systems use the frequency range of 0.2 to $0.6 \mathrm{MHz}$ to evaluate BUA. ${ }^{(21)}$

BUA provides good assessment of bone mass as strong correlation has been observed between BMD and BUA. Laugier and colleagues observed statistically significant relationship between normalized BUA and volumetric bone mineral density (vBMD) with $R^{2}$ of 0.8 and pvalue less than $10^{-4}$ upon the measurement of the excised trabecular bone from the calcaneus using a water-based QUS system. ${ }^{(22)}$

Wide range of BUA values has been reported in studies of the human calcaneus. In study by Portero and colleagues, which assessed the excised calcaneus using Hologic Sahara ${ }^{\circledR}$ QUS device, reported average BUA of $38.2 \mathrm{~dB} / \mathrm{MHz}$ with wide range of BUA observed between 0.3 and 80.1 $\mathrm{dB} / \mathrm{MHz} .{ }^{(23)}$ In study by Bouxsein and colleagues, which evaluated the calcaneus from intact feet of cadavers using Hologic UBA575+ ${ }^{\circledR}$ QUS device, average BUA of $53.6 \mathrm{~dB} / \mathrm{MHz}$ with BUA range of 8.0 to $92.5 \mathrm{~dB} / \mathrm{MHz}$ were observed. ${ }^{(24)}$ The observed difference between the two studies 
can be attributed to the difference in the QUS systems used for the assessment, and the difference in the calcaneus condition (ex vivo calcaneus vs. calcaneus from intact feet).

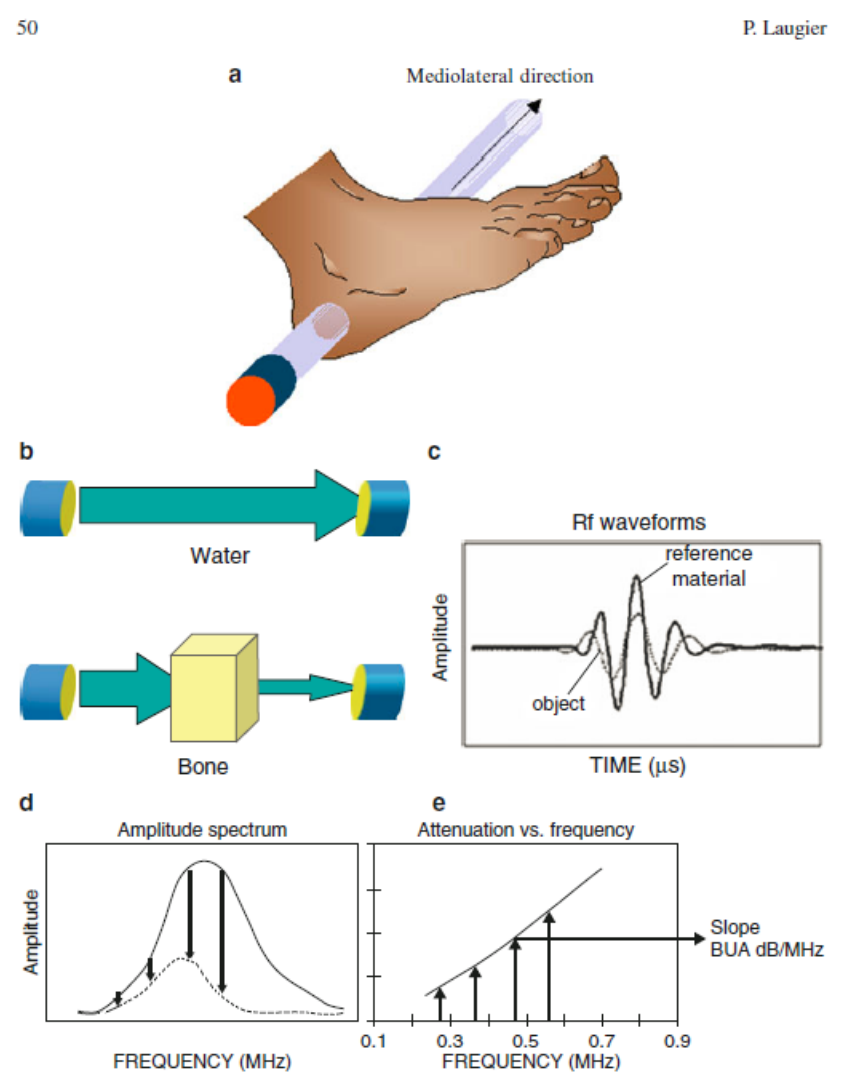

Fig. 1.1. Principles of ultrasound transverse transmission at the calcaneus to measure BUA. (a) Placement of transducers in the mediolateral direction, (b) substitution method of assessing BUA, (c) difference in the amplitude signal obtained from the reference medium (solid line) and through the heel (dashed line), (d) difference in the amplitude spectra of the reference signal (solid line) and of the signal transmitted through the heel (dashed line) (e) frequency dependent

$$
\text { attenuation }^{(15)}
$$

\subsubsection{Speed of Sound}

Speed of sound is a characteristic of a medium, which depends on the material and geometric properties and temperature of the medium. Generally, speed of sound, $c$, can be derived from an 
effective elastic modulus $M_{e}$ and effective mass density $\rho_{e}$ as per Equation 1.15 . The effective elastic modulus and effective mass density account for different wave types such as bulk compression, bulk shear, surface or guided wave. ${ }^{(19)}$

$$
c=\sqrt{\frac{M_{e}}{\rho_{e}}}
$$

However, the derivation of $M_{e}$ for the bone is complex and requires cumbersome theoretical developments. It is because the bone is a highly heterogeneous medium containing a mixture of components such as collagen fibers, hydroxyapatite crystals, water, and bone marrow which have different elastic coefficients. ${ }^{(15)}$ Furthermore, because the pore size $(\sim 500$ to $1000 \mu \mathrm{m})$ of the trabecular bone is comparable to the wavelength $(1.5 \mathrm{~mm}$ at $500 \mathrm{kHz})$, the trabecular bone cannot be approximated to be homogeneous. ${ }^{(15)}$

QUS systems evaluate SOS based on time of flight (TOF) measurements. TOF represents the time delay between the initial transmission of ultrasound from one transducer and reception by another. For the water-based system, two TOF measurements are taken in order to derive SOS. Initially, TOF of an ultrasound pulse without a sample is measured through a reference medium (water) with length $L$ between the two transducers. TOF of the reference medium can be expressed using $L$ and the temperature dependent speed of sound, $c^{\text {ref }}$, in the reference medium:

$$
T O F^{r e f}=\frac{L}{c^{r e f}}
$$

Then TOF of ultrasound with a sample of thickness $x$ in between the transducer is then measured as:

$$
T O F=\frac{L-x}{c^{r e f}}+\frac{x}{c}
$$

The difference of TOF in the two conditions can be written as:

$$
\Delta T O F=T O F-T O F^{r e f}=\frac{x}{c}-\frac{x}{c^{r e f}}
$$


Based on Equation 1.18, SOS in the target sample can be calculated as:

$$
c=\frac{1}{\left(\frac{1}{c^{r e f}}\right)+\left(\frac{\Delta T O F}{x}\right)}
$$

In case of a dry contact QUS system, whose transducers are in direct contact with the sample, SOS is derived by dividing mechanically measured thickness $x$ with TOF.

$$
c=\frac{x}{T O F}
$$

Similar to BUA, SOS exhibits strong correlation with bone mass density. In the study by Laugier and colleagues on the excised trabecular bone of the calcaneus, statistically significant relationship between SOS and vBMD was reported $\left(R^{2}=0.84\right.$, and $\left.\mathrm{p}<10^{-4}\right) .^{(22)}$ In addition, statistically significant relationship was observed between normalized BUA and SOS as well $\left(R^{2}\right.$ $=0.88$, and $\left.\mathrm{p}<10^{-4}\right)$. The mean SOS in bone measured by Portero and colleagues is $1571 \mathrm{~m} / \mathrm{s}$ $(1412-1746 \mathrm{~m} / \mathrm{s})$ for the excised calcaneus, ${ }^{(24)}$ and mean value measured by Bouxsein and colleagues is $1512 \mathrm{~m} / \mathrm{s}(1480-1570 \mathrm{~m} / \mathrm{s})$ for the intact foot.

\subsubsection{Stiffness Index}

Stiffness index (SI), also known as quantitative ultrasound index (QUI), is an empirical parameter derived from the combination of BUA and SOS. It is important that the term SI should not be confused with the mechanical stiffness of bone. The empirical derivation of SI is specific to each device and the rationale for the specific derivation equation remains proprietary to the manufacturer. The equation used by GE Lunar Achilles ${ }^{\circledR}$ and Hologic Sahara ${ }^{\circledR}$ QUS devices to derive SI can be expressed as Equation $1.21^{(25)}$ and $1.22,{ }^{(26)}$ respectively.

$$
\begin{aligned}
& \mathrm{SI}=0.67 \times(\mathrm{BUA})+0.28 \times(\mathrm{SOS})-420 \\
& \mathrm{SI}=0.41 \times(\mathrm{BUA}+\mathrm{SOS})-571
\end{aligned}
$$

SI has been reported to produce better precision than using BUA or SOS alone, because it compensates for both variation in calcaneal thickness and temperature. ${ }^{(27)} \mathrm{Xu}$ and colleagues 
observed significantly positive correlation between calcaneal SI and total body aBMD $\left(R^{2}=0.693\right.$, $\mathrm{p}<0.001) .{ }^{(28)}$ To facilitate direct comparison with DXA, SI can be converted to give estimated aBMD based on SI T-score. ${ }^{(29)}$

\subsection{Bone Seeking Elements}

Bone-seeking elements are class of chemical elements that are incorporated into crystalline bone matrix with extended residence, whose whole-body kinetic behavior is determined by the balance among excretion, bone uptake and release from bone. ${ }^{(30)}$ Bone-seeking elements such as strontium, lead, aluminum, uranium and barium have significant physiological implication as they can alter bone tissue or its growth rate.

\subsubsection{Strontium}

Strontium $(\mathrm{Sr}, \mathrm{Z}=38)$ is an alkaline earth metal positioned directly one row under calcium in the periodic table. It holds clinical significance due to its role as a medication for osteoporosis treatment. Strontium renalate is orally administered drug licensed for the treatment of postmenopausal osteoporosis in Europe and Australia. ${ }^{(31)}$ Although strontium ranelate is not yet approved for the medicinal use in the United States or Canada, other forms of strontium salts such as strontium citrate and strontium carbonate are commercially available as over-the-counter nutritional supplements. ${ }^{(32)}$

The therapeutic effect of increase in bone mass observed from the intake of strontium medication is due to its dual effect on bone remodelling: promotion of bone formation and inhibition of bone reabsorption. ${ }^{(33)}$ The promotion of bone formation is achieved by anabolic effect of increasing pre-osteoblast replication and osteoblast differentiation, while the inhibition of bone reabsorption is due to inhibition of osteoclast differentiation and enhanced osteoclast apoptosis. 
The clinical efficacy of strontium treatment has been documented in large population studies such as Spinal Osteoporosis Therapeutic Intervention (SOTI) study ${ }^{(31)}$ and Treatment of Peripheral Osteoporosis (TROPOS) study. ${ }^{(34)}$ In SOTI study, aBMD increased by $14.4 \%$ and $8.3 \%$ in the lumbar spine and femoral neck, respectively, after 36 months of daily intake of $2 \mathrm{~g}$ strontium renalate among 1648 women. Similarly, TROPOS study reported $8.2 \%$ and $9.8 \%$ increase respectively in aBMD of the femoral neck and the total hip after 3 years of daily intake of $2 \mathrm{~g}$ strontium renalate among 5091 women. Upon the chemical analysis of the iliac bone biopsy samples, average bone strontium content (expressed as $\mathrm{Sr} /[\mathrm{Sr}+\mathrm{Ca}], \mathrm{mol} / \mathrm{mol} \%$ ) of $1.6 \%$ was observed among patients who underwent strontium renalate treatment for three years. ${ }^{(35)}$

However, the increase in aBMD measured by DXA in the presence of strontium is partially an artifact caused by increased attenuation coefficient in strontium containing bone. ${ }^{(36,37)}$ Because strontium has higher attenuation coefficient than calcium in the diagnostic X-ray energy range, strontium containing bone would attenuate more photon and as the result aBMD measured by DXA is overestimated. Nielsen and colleagues ${ }^{(37)}$ reported strontium overestimation factor, which is the degree of aBMD overestimation observed per $1 \%$ increase in bone strontium content, to be $10 \%$. The overestimation can be expressed as following equation ${ }^{(38)}$ :

$$
B M D_{\text {measured }}=(C \times S r \%+1) B M D_{\text {Actual }}
$$

where $C$ denotes the overestimation factor, $S r \%$ denotes the strontium ratio. Liao and colleagues further investigated the overestimation factor and reported the variation in the overestimation factor among different DXA devices. ${ }^{(39)}$ This is because the energy level of X-ray beams used by different DXA manufacturers varies, and in turn the variation in energy dependent attenuation coefficient affects the overestimation factor. Therefore, in order to accurately investigate the 
therapeutic effect of strontium, the bone mass needs to be assessed independent from the strontium content.

\subsubsection{Lead and Aluminum}

Lead $(\mathrm{Pb}, \mathrm{Z}=82)$ is another bone-seeking element and $90 \%$ of the body burden of lead is deposited in bone. ${ }^{(40)}$ In addition to the neurotoxicity, lead cause degeneration of bone mineral and consequently, decrease in aBMD measured by DXA. ${ }^{(41,42)}$ Lead inhibits both osteoblast and osteoclast activity, but the inhibitory effect is more pronounced in osteoblast than osteoclast. ${ }^{(43)}$ As a result, bone reabsorption becomes more dominant than bone formation in the process of bone turnover. The major pathway of lead ingestion is occupation exposure in mines and smelters. ${ }^{(44,45)}$ In addition, environmental exposure from lead piping and lead based paint contribute to the lead ingestion of general public. ${ }^{(46)}$ The accumulation of lead in bone occurs in relatively low concentration in comparison to strontium, as the exposure to lead is inadvertent and not deliberate. In extreme case of an occupational exposure in Silesian district of Poland, lead concentration ranged from $20 \mu \mathrm{g} / \mathrm{g}$ to $200 \mu \mathrm{g} / \mathrm{g}$ bone weight. ${ }^{(45)}$

Aluminum $(\mathrm{Al}, \mathrm{Z}=13)$ is a toxic bone-seeking element and about $60 \%$ of the aluminum body burden is deposited in bone. ${ }^{(47)}$ The toxicity of aluminum has been linked to Alzheimer's disease $^{(48)}$ and osteomalacia, which is softening of bone. ${ }^{(49)}$ Aluminum toxicity to bone can be attributed to its inhibitory effects on osteoblastic activity, which results in diminished collagen synthesis and matrix mineralization. ${ }^{(49)}$ Aluminum can be introduced into human body via occupational exposure $^{(48)}$ and aluminum containing medication or parenteral nutrition. ${ }^{(50-52)}$ Furthermore, chronic exposure to aluminum via consumption of food and consumer products is of concern. ${ }^{(53,54)}$ Similar to lead, the concentration of aluminum accumulated in bone is lower than 
strontium; extreme cases of aluminum contamination among patients undergoing hemodialysis did not exceed $130 \mu \mathrm{g} / \mathrm{g}^{(55)}$

Because lead is a highly attenuating element, elevation of aBMD due to lead is of a potential concern. ${ }^{(43,56)}$ Two distinct studies have been conducted to investigate the effect of lead on aBMD measured by DXA. Puzas and colleagues examined pulverized bovine bone doped with lead using a pencil beam DXA device (Lunar DPX-L ${ }^{\circledR}$ ) and observed 4 to $11 \%$ increase in aBMD for lead concentration ranging from 10 to $100 \mu \mathrm{g} / \mathrm{g} .{ }^{(56)}$ In contrast, the overestimation of aBMD was not observed in another study done by Popovic and colleagues that measured plaster of Paris phantoms doped with clinically relevant concentration of lead using fan beam DXA device (Hologic QDR $\left.4500 \mathrm{~A}^{\circledR}\right)$. It appears that, to date, no scientific study has investigated the effect of clinically relevant concentration of aluminum on aBMD.

\subsection{Bone-Mimicking Phantoms}

Various models of trabecular bone-mimicking phantoms for QUS systems have been proposed previously. Clarke and colleagues ${ }^{(57)}$ developed an epoxy resin based phantom and the porosity of the trabecular bone was simulated using granules of porcine gelatin that mimics bone marrow. This model of phantom is often called the Leeds phantom. Hodgskinson and colleagues $^{(58)}$ developed a phantom made of a Perspex block, where a symmetrical array of holes were introduced to simulate the porosity of the trabecular bone; the number of the holes were kept constant and the porosity was controlled by changing the size of the holes. For both models of bone-mimicking phantom, non-linear parabolic relationship was observed between BUA and the

porosity. ${ }^{(57,58)}$ In addition, Tatarinov and colleagues ${ }^{(59)}$ further modified the Leeds phantom with porosity simulation using soft rubber particles instead of gelatin granules, and imitated bone 
mineral content by adding the mineral residue of natural bone obtained by grinding and burning. Other models that have been proposed for trabecular bone-mimicking phantom include aluminum foams ${ }^{(60)}$ nylon filaments suspended in soft-tissue mimicking material ${ }^{(61)}$ and Polyacetal cuboid with cylindrical pores. ${ }^{(62)}$

Da Silva and colleagues developed protocols for the synthesis of pure hydroxyapatite phantom free of strontium or lead contamination ${ }^{(63)}$ and for the synthesis of strontium substituted hydroxyapatite phantom with high strontium concentrations. ${ }^{(64)}$ Rizivi and colleagues ${ }^{(65,66)}$ developed a QUS compatible trabecular bone-mimicking phantom made of the hydroxyapatite synthesized using the above methods; the hydroxyapatite phantoms are finely milled and mixed with gelatin medium homogeneously. The gelatin based bone-mimicking phantom is housed in an acrylic box and is submerged in castor oil that mimics soft tissues overlying the calcaneus. SOS and BUA measured from the multi-layer bone-mimicking phantom were similar to the acoustic parameters measured from the ex vivo trabecular bone from the human calcaneus. ${ }^{(23)}$

\subsection{Hypothesis and Specific Aims}

DXA technique, which is the current gold standard of bone densitometry, relies on X-ray attenuation to measure BMD. Consequently, the presence of bone-seeking elements such as strontium, aluminum and lead could cause systematic error in DXA-based BMD measurements. In contrast, QUS technique measures BMD based on bone's macroscopic acoustic properties, which are not influenced by microscopic changes in the atomic composition of the bone. Therefore, it is hypothesized that QUS measurements of BMD would remain independent of concentration of these bone-seeking elements. This study aims to investigate the effect of clinically relevant concentrations of strontium, lead and aluminum on DXA and QUS bone 
densitometry measurements using custom-designed bone-mimicking phantoms. The specific aims of this study are:

- $\quad$ Producing bone-mimicking phantoms compatible with DXA and QUS systems that contain known concentrations of strontium, lead and aluminum, while maintaining constant vBMD.

- Verifying that the bone-mimicking phantoms correctly reproduce the acoustic parameters measured from the calcaneus and yield desired aBMD under DXA measurements.

- Investigating the impact of the clinically relevant concentrations of strontium, lead and aluminum in the bone-mimicking phantom on aBMD measured by DXA and the acoustic parameters measured by QUS. 


\section{CHAPTER 2:ASSESSMENT OF THE EFFECT OF STRONTIUM, LEAD AND ALUMINUM ON DUAL-ENERGY X-RAY ABSORPTIOMETRY AND QUANTITATIVE ULTRASOUND MEASUREMENTS USING TRABECULAR BONE-MIMICKING PHANTOMS}

This chapter presents a manuscript that was submitted to Medical Physics, the

International Journal of Medical Physics Research and Practice.

Manuscript \#: 16-1416

Submission Date: September 16, 2016 


\title{
Assessment of the Effect of Strontium, Lead and Aluminum on \\ Dual-Energy X-Ray Absorptiometry and Quantitative Ultrasound Measurements
}

Using Trabecular Bone-Mimicking Phantoms

\author{
Deok Hyun Jang, Eric Da Silva and Ana Pejović-Milić a) \\ Department of Physics, Ryerson University, Toronto, Ontario, M5B 2K3, Canada
}

\begin{abstract}
Jahan Tavakkoli
Department of Physics, Ryerson University, Toronto, Ontario, M5B 2K3, Canada; and Institute for Biomedical Engineering, Science and Technology (iBEST), Keenan Research Centre for Biomedical Science, St. Michael's Hospital, Toronto, Ontario, M5B 1T8, Canada
\end{abstract}

Lubomira Slatkovska and Angela M. Cheung Centre of Excellence in Skeletal Health Assessment, Joint Department of Medical Imaging, University of Toronto, Toronto, Ontario, M5G 2C4, Canada; and Osteoporosis Program, University Health Network, Toronto, Ontario, M5G 2C4, Canada

a) Author to whom correspondence should be addressed. Electronic mail: anamilic@ ryerson.ca

Purpose: Dual-energy X-ray absorptiometry (DXA) is the gold standard technique to measure areal bone mineral density (aBMD) for the diagnosis of osteoporosis. Because DXA relies on the attenuation of photon to predict aBMD, deposition of bone-seeking elements such as strontium, lead and aluminum that differ in atomic numbers from calcium can cause inaccurate estimation of aBMD. Quantitative ultrasound (QUS), is another technique available to assess bone health by 
measuring broadband ultrasound attenuation (BUA), speed of sound (SOS) and an empirically derived quantity called stiffness index (SI). Because the acoustic properties are not prone to significant change due to microscopic changes in the atomic composition of bone, it is hypothesized that QUS is unaffected by the presence of bone-seeking elements in bone. The objective of this study was to investigate the effect of strontium, lead and aluminum on aBMD measured by DXA and the acoustic parameters measured by QUS using trabecular bonemimicking phantoms compatible with both techniques.

Methods: Trabecular bone-mimicking phantoms were produced by homogeneously mixing finely powdered hydroxyapatite compounds that contain varying concentrations of strontium, lead or aluminum with porcine gelatin solution. Seven strontium-substituted phantoms were produced with varying molar ratio of $\mathrm{Sr} /(\mathrm{Sr}+\mathrm{Ca})$ ranging from 0 to $2 \%$. Four lead-doped phantoms and four aluminum-doped phantoms were constructed with the respective analyte concentrations ranging from 50 to $200 \mathrm{ppm}$. An additional $0 \mathrm{ppm}$ phantom was produced to be used as a baseline for the lead and aluminum phantom measurements. All phantoms had uniform volumetric bone mineral density (vBMD) of $200 \mathrm{mg} / \mathrm{cm}^{3}$, and were assessed using a Hologic Horizon ${ }^{\circledR}$ DXA device, a Hologic Sahara ${ }^{\circledR}$ QUS device, and an in-house research QUS system.

Results: Direct linear relationship was found between aBMD measured by DXA and strontium concentration $(p<0.001)$. From the measurement of lead and aluminum using DXA, statistically significant negative relationship ( $p=0.016, r=-0.347$ and $p<0.001, r=-0.549$, respectively) were observed between aBMD and the analyte concentrations. No statistically significant change in measured SI values with respect to the concentration of all three elements was observed, for both the clinical and research QUS systems. 
Conclusion: aBMD measured by DXA is prone to overestimation in the presence of strontium, but acoustic parameters measured by QUS are independent of strontium concentration. The deviation in aBMD induced by lead concentration under $200 \mathrm{ppm}$ and aluminum concentration under 100 ppm cannot be detected using Hologic Horizon ${ }^{\circledR}$ DXA device, as the changes were within $1 \%$ coefficient of variation of the device. Furthermore, the SI measured by the QUS systems are not affected by lead or aluminum concentrations used in this study.

Key words: DXA, Bone Quantitative Ultrasound, Osteoporosis, Densitometry, Bone-seeking elements

\subsection{Introduction}

Dual-energy X-ray absorptiometry (DXA) is the current gold standard method for the diagnosis of osteoporosis and the monitoring of treatment progress. ${ }^{(17)}$ DXA estimates areal bone mineral density (aBMD) by measuring the transmission ratio of two X-ray beams with different photon energies. ${ }^{(9)}$ The mass attenuation coefficient, which in turn determines the transmission ratio of the two beams, is energy and atomic number dependent. Therefore, change in the atomic composition of bone results in change in the overall mass attenuation of the tissue. ${ }^{(36)}$ One of the main limitations of DXA is that deposition of bone-seeking elements, which differ in atomic numbers from calcium $(\mathrm{Ca}, \mathrm{Z}=20)$, can cause inaccurate estimation of aBMD. ${ }^{(36)}$

A Bone-seeking element can be defined as any element that is readily incorporated into bone with extended residence time by substituting calcium. ${ }^{(30)}$ Examples of bone-seeking elements are strontium, lead, aluminum, radium, uranium and barium. Elements that have a higher atomic number than calcium, such as strontium $(\mathrm{Sr}, \mathrm{Z}=38)$ and lead $(\mathrm{Pb}, \mathrm{Z}=82)$, have higher mass 
attenuation coefficients than calcium at photon energy level relevant for DXA. Therefore, such elements are expected to increase photon attenuation in bone and result in the overestimation of aBMD. ${ }^{(36)}$ In contrast, it is anticipated that elements such as aluminum ( $\left.\mathrm{Al}, \mathrm{Z}=13\right)$, which have lower mass attenuation coefficients than calcium, may cause underestimation of aBMD as assessed by DXA.

Strontium is a bone-seeking element of clinical importance, as strontium ranelate is used as an orally administered medication to treat osteoporosis ${ }^{(31)}$ presently approved in Europe and Australia. Although strontium ranelate is not licenced in the United States or Canada, other strontium salts such as strontium citrate and strontium carbonate is commercially available as an over-the-counter nutritional supplement. ${ }^{(32)}$ Strontium has the therapeutic effect of increasing bone mass by stimulating osteoblasts and inhibiting osteoclasts. ${ }^{(33)}$ Moreover, reduction in the risk of fracture among postmenopausal women has been reported by the Spinal Osteoporosis Therapeutic Intervention (SOTI) ${ }^{(31)}$ and Treatment of Peripheral Osteoporosis (TROPOS) trials. ${ }^{(34)}$ However, in addition to the inherent therapeutic effect of strontium, the increase in aBMD is partially due to the increase in photon attenuation. ${ }^{(38)}$ The overestimation of aBMD due to strontium has been demonstrated by several in vitro studies involving DXA measurements of hydroxyapatite with partial strontium substitution. ${ }^{(36,37,39)}$ The study by Nielsen et al. demonstrated approximately $10 \%$ aBMD overestimation for $1 \mathrm{~mol} / \mathrm{mol} \%$ of $\mathrm{Sr} /(\mathrm{Sr}+\mathrm{Ca}) .{ }^{(37)}$

Contrary to the therapeutic effect of strontium, lead and aluminum are toxic elements that may cause degeneration of the bone matrix..$^{(41,42,49,67)}$ The pathways of lead intake include occupational exposure in industry, ${ }^{(41,45)}$ and environmental exposure from lead pipes and lead based paint. ${ }^{(46)}$ Aluminum can be introduced to the human body through occupational exposure, and historically it tended to be introduced through the ingestion of aluminum-based medications 
for renal failure. ${ }^{(50,52)}$ However, concentrations of the two elements observed in bone tends to be significantly lower than that of strontium, due to the toxicity of lead and aluminum. ${ }^{(45,47,55)}$ To date, there are two studies ${ }^{(43,56)}$ investigating the effect of low levels of lead on aBMD in two different samples: each with conflicting results. Puzas and colleagues measured aBMD of pulverized bovine bone doped with lead and observed $4-11 \%$ overestimation for lead concentration ranging from 10 to $100 \mu \mathrm{g}$ lead/g bone. ${ }^{(56)}$ In contrast, Popovic and colleagues ${ }^{(43)}$ measured aBMD of plaster of Paris bone phantoms doped with lead and observed no statistically significant aBMD deviation. To date, it appears that the effect of aluminum on aBMD has not yet been demonstrated by scientific studies.

Quantitative Ultrasound (QUS) is a relatively inexpensive, portable and non-ionizing technique $^{(18)}$ that offers an alternative means of assessing bone health. Conventional clinical QUS devices measure broadband ultrasound attenuation (BUA) and speed of sound (SOS) at the calcaneus bone site. Stiffness index (SI), an empirically defined quantity established to compensate for the variation in the calcaneal thickness and temperature, ${ }^{(27)}$ is derived from the linear combination of the two acoustic parameters. Clinical studies have demonstrated moderately positive correlation for aBMD measured at the lumbar spine and the femoral neck, with SOS, BUA and SI measured by calcaneal QUS. ${ }^{(28,68-71)}$ Consequently, calcaneal QUS can effectively identify postmenopausal women at risk of bone fracture. ${ }^{(72,73)}$

Our research group has previously developed a bone-mimicking phantom by synthesizing pure hydroxyapatite without contamination of strontium and lead ${ }^{(63)}$ and hydroxyapatite with controlled substitution of strontium. ${ }^{(64)}$ More recently, Rizvi and colleagues ${ }^{(65,66)}$ further developed a trabecular bone-mimicking phantom for QUS measurements, by suspending the finely powdered hydroxyapatite homogeneously in gelatinous medium. 
The objective of this study was to investigate if the presence of clinically relevant concentrations of strontium, lead or aluminum in bone induce statistically significant deviation in DXA and QUS bone constructed measurements. The bone-mimicking phantoms with uniform volumetric bone mineral densities (vBMD) were constructed using hydroxyapatite with varying concentrations of strontium, lead or aluminum and were measured using Hologic Horizon ${ }^{\circledR}$ DXA device, Hologic Sahara ${ }^{\circledR}$ QUS device and an in-house research QUS system.

\subsection{Materials and Methods}

\subsubsection{Hydroxyapatite-based trabecular bone-mimicking phantom}

The hydroxyapatite bone phantoms were prepared using the method developed by Da Silva and colleagues. ${ }^{(63,64)}$ The strontium-substituted hydroxyapatite bone phantoms were produced by mixing $\mathrm{CaHPO}_{4} \cdot 2 \mathrm{H}_{2} \mathrm{O}, \mathrm{Ca}(\mathrm{OH})_{2}$ and $\mathrm{Sr}(\mathrm{OH})_{2} \cdot 8 \mathrm{H}_{2} \mathrm{O}$, while maintaining a constant $(\mathrm{Ca}+\mathrm{Sr}) / \mathrm{P}$ molar ratio of 1.67 and thus producing a mineral phase with the formula of $\mathrm{Ca}_{5-\mathrm{x}} \mathrm{Sr}_{\mathrm{x}}\left(\mathrm{PO}_{4}\right)_{3} \mathrm{OH} .{ }^{(64)}$ The hydroxyapatite bone phantoms doped with lead or aluminum were synthesized by mixing $\mathrm{CaHPO}_{4} \cdot 2 \mathrm{H}_{2} \mathrm{O}$ and $\mathrm{Ca}(\mathrm{OH})_{2}$ with constant $\mathrm{Ca} / \mathrm{P}$ molar ratio of 1.67 and adding $1000 \mu \mathrm{g} / \mathrm{mL}$ lead or aluminum standard solution to the mixture in the liquid phase to achieve desired concentration of the analytes. The phantoms were set by adding $1 \mathrm{M} \mathrm{Na}_{2} \mathrm{HPO}_{4}$ setting solution to the powdered mixture at a powder-to-liquid ratio of powder-to-liquid of 2:1.

The protocol developed by Rizvi and colleagues ${ }^{(65,66)}$ was used to produce the gelatin-based trabecular bone phantoms. Once the hydroxyapatite phantoms were set, they were dried to constant weight at $80{ }^{\circ} \mathrm{C}$ and then were finely powdered using a tungsten carbide ball mill. The powdered phantoms were added to $5 \% \mathrm{w} / \mathrm{w}$ porcine gelatin solution to achieve a mineral density of $200 \mathrm{mg} / \mathrm{cm}^{3}$, similar to the mean vBMD $\left(213 \mathrm{mg} / \mathrm{cm}^{3}\right)^{(22)}$ of trabecular bone of the calcaneus 
measured by QCT. ${ }^{(22)}$ The gelatin mixture was poured into a container with dimensions of $6.5 \times$ $6.5 \times 2.5 \mathrm{~cm}^{3}$ for molding. The phantom width of $2.5 \mathrm{~cm}$ was chosen to represent the thickness of the human calcaneus. ${ }^{(20)}$ The container was rotated for two hours to ensure homogeneous distribution of the hydroxyapatite particles within the gelatin medium and to prevent aggregation of the particles. The phantoms were cooled down in a refrigerator at $4{ }^{\circ} \mathrm{C}$ for 12 hours to allow solidification of the gelatin medium, and once solidified, the phantoms were removed from the container.

Seven strontium-substituted phantoms were constructed with the $\mathrm{mol} / \mathrm{mol}[\mathrm{Sr} /(\mathrm{Sr}+\mathrm{Ca})] \%$ ranging from 0 to $2 \%$. This range of the strontium concentrations was chosen to reflect the biologically relevant concentrations observed after prolonged intake of strontium ranelate. ${ }^{(74,75)}$ For lead and aluminum, four phantoms for each element were constructed with the analyte concentration ranging from 50 to $200 \mathrm{ppm}$ (mass of analyte/mass of hydroxyapatite). This range of concentration of the analytes was chosen to include the concentrations observed in extreme cases of prolonged exposure to lead ${ }^{(45)}$ and aluminum. ${ }^{(55)}$ An additional phantom that does not contain any analyte was constructed as a reference phantom to be used for both lead and aluminum study. Besides QUS, the trabecular bone-mimicking phantoms are compatible with DXA as well, since the atomic composition of hydroxyapatite mimics the bone mineral content of human bone.

\subsubsection{DXA Measurements}

DXA measurements of the trabecular bone mimicking phantoms were conducted using a Hologic Horizon ${ }^{\circledR}$ device (Hologic Inc., Bedford, MA, USA). Hologic Horizon ${ }^{\circledR}$ have operating potentials of $100 \mathrm{kVp}$ and $140 \mathrm{kVp}$, resulting in two X-ray spectra with low and high energy. The trabecular bone-mimicking phantoms were placed in a water bath to simulate overlying soft tissue present during a human calcaneus measurement. The overall depth of the water bath was 
maintained as $4 \mathrm{~cm}$, so that the layer of water above the phantom has a height of $1.5 \mathrm{~cm}$ (Fig. 2.1). The $1.5 \mathrm{~cm}$ of overlying layer of water represents the overall thickness of the soft tissue overlying the calcaneus. ${ }^{(76,77)}$ Because the Hologic Horizon ${ }^{\circledR}$ system did not have the BMD analysis protocol available for the calcaneus, the lumbar spine protocol was used for the BMD analysis of the phantoms. Each phantom was measured ten times with repositioning between measurements. The region of interest (ROI) was kept constant throughout all measurements, as the size of ROI can influence the result of BMD analysis.

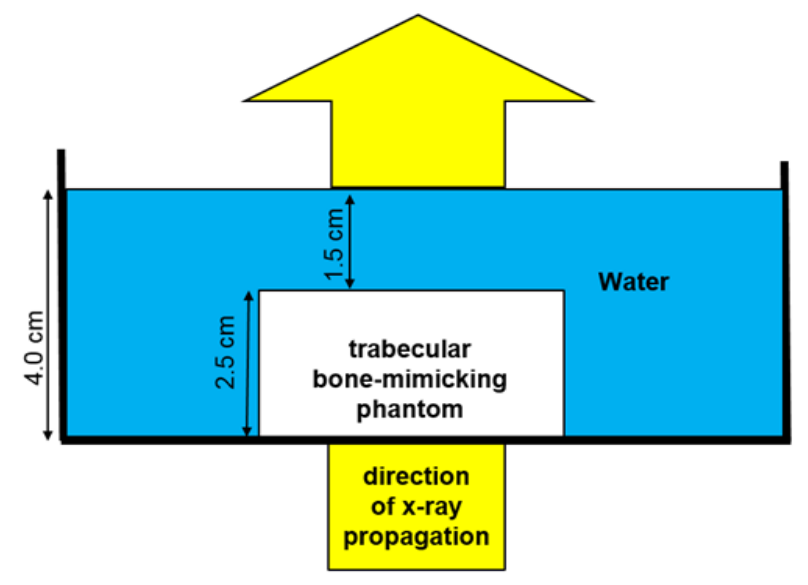

Fig. 2.1. Schematic experimental setup of the DXA measurements

\subsubsection{Clinical QUS Measurement}

The clinical QUS measurements were performed using a Hologic Sahara ${ }^{\circledR}$ device (Hologic Inc., Bedford, MA, USA). Because the two ultrasound transducers of the QUS system apply mechanical pressure that can compress and thus damage the gelatin-based phantom, a multi-layer bone phantom was developed by Rizvi and colleagues ${ }^{(65)}$ to protect the gelatin-based phantom from the pressure. The phantom was housed in an acrylic box with a thin Mylar window to allow the transmission of ultrasound. The gelatin-based phantom was then submerged in castor oil to remove the air gap and to simulate the soft tissue overlying the calcaneus bone site. The thickness 
of the castor oil layer on each side of the phantom was $0.75 \mathrm{~cm}$, which represents the thickness of the soft tissue on the lateral and medial sides of the calcaneus (Fig. 2.2) ${ }^{(76,77)}$

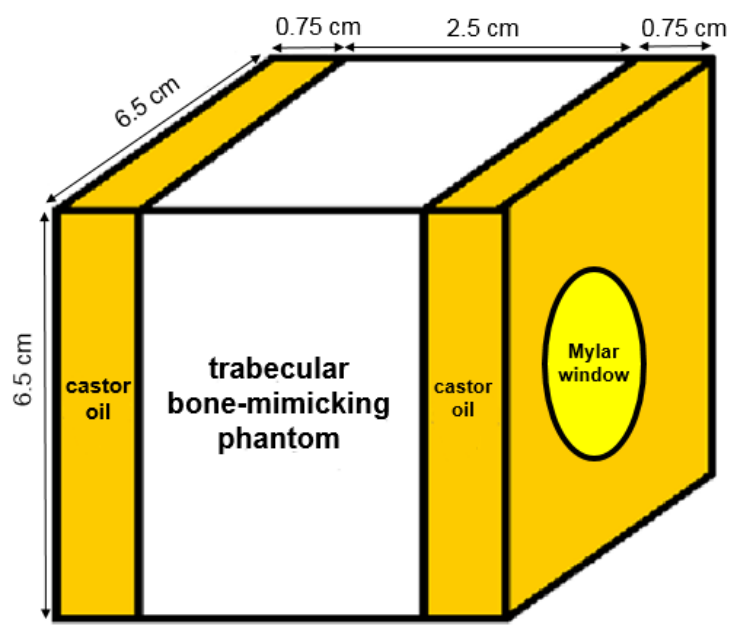

Fig. 2.2. Schematic design of the multi-layer trabecular bone-mimicking phantom that mimics bone and overlying soft tissue at the calcaneus bone site

The multi-layer phantom was placed in between the two transducers of the Sahara ${ }^{\circledR}$ QUS device, and petroleum gel was applied on the transducers to create optimal contact with the surface of the phantom. Each constructed phantom was five times for SOS, BUA and SI with the repositioning of the phantom in between each measurement. The device-specific formula used to derive SI is given below: ${ }^{(26)}$

$$
\mathrm{SI}=0.41(\mathrm{SOS}+\mathrm{BUA})-571
$$

\subsubsection{In-house Research QUS Measurements}

An in-house research QUS system was available in our laboratory to investigate the proof of principle of QUS, and to verify the results from the clinical device. ${ }^{(65)}$ The research system consisted of a transducer connected to a function generator that serves as the transmitter and another identical transducer connected to a digital oscilloscope that serves as a receiver. The 
specifications for the two transducers were as following: central frequency $=1 \mathrm{MHz}$, focal length $=10.1 \mathrm{~cm}$ and aperture diameter $=3.0 \mathrm{~cm}$. The pair of transducers were coaxially and confocally aligned to maximize the signal amplitude detected by the receiver. The transducers were immersed in degassed and deionized water and the multi-layer phantom was placed halfway between the transducers and centred at the location of the common focal point (Fig. 2.3).

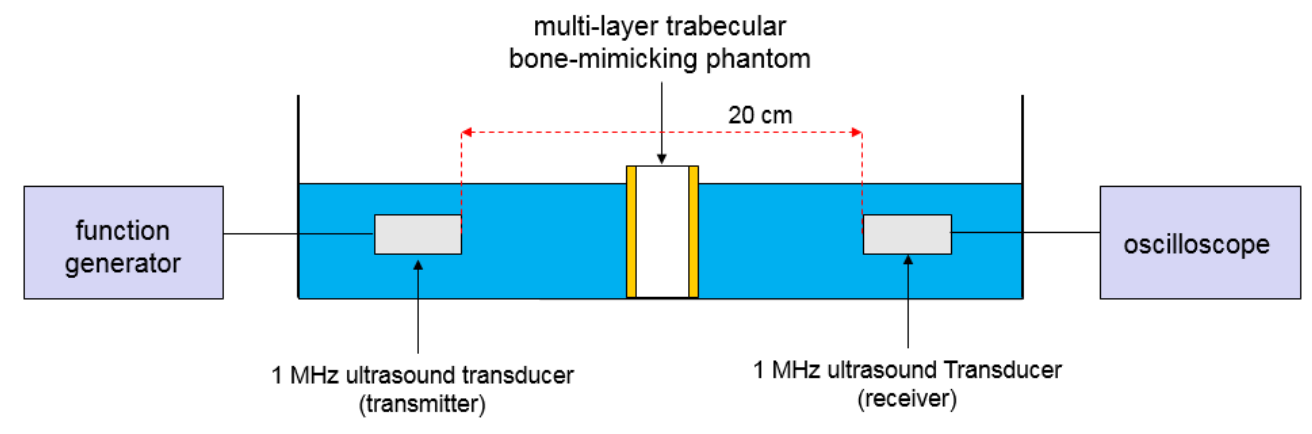

Fig. 2.3. Schematic experimental set up of the in-house research QUS system

Five measurements of BUA, SOS and SI were taken for each phantom with repositioning of the phantom in between each measurement. Attenuation of ultrasound in the multi-layer phantom was measured over the frequency range of 0.5 to $1.4 \mathrm{HMz}$. Then BUA with the units of $\mathrm{dB} / \mathrm{MHz}$ was determined from the slope of the linear regression of the measured attenuation versus frequency. In order to obtain SOS, two time-of-flight (TOF) measurements of $1 \mathrm{MHz}$ ultrasound signal were performed with and without the phantom. Equation 2.2 was used to calculate SOS based on the difference in time-of-flight $(\triangle \mathrm{TOF})$ between the two measurements: ${ }^{(15)}$

$$
\mathrm{SOS}=\frac{1}{\frac{1}{c_{\text {ref }}}+\frac{\Delta \mathrm{TOF}}{\mathrm{L}}}
$$

where $c_{r e f}$ is the speed of sound in deionized water, which is the reference medium, and L is the thickness of the phantom, which was $4 \mathrm{~cm}$. As the research QUS system does not have its own 
formula for SI derivation, the SI value for the research system was derived from BUA and SOS values using Equation 2.1 to allow the direct comparison of the parameter with that of the clinical QUS.

\subsubsection{Statistical Analysis}

The statistical analysis was conducted with IBM SPSS (version 23). Linear regression analysis was performed to assess the relationship between the analyte concentration and the parameters measured using DXA and both QUS systems. The Pearson's correlation coefficients $(r)$ and linear regression coefficient, which was the slope of the linear regression, were calculated for the evaluation of possible correlations. A p-value less than 0.05 was considered statistically significant, thus statistical evaluations were made at the $95 \%$ level of confidence.

\subsection{Results}

\subsubsection{Strontium Phantom Measurements}

Assessment of the reference phantom containing 0\% strontium by the Hologic Horizon ${ }^{\circledR}$ DXA device demonstrated a satisfactory level of accuracy. Based on the phantom dimension and hydroxyapatite content, the expected measurement results for area, bone mineral content (BMC) and BMD were $42.25 \mathrm{~cm}^{2}, 21.125 \mathrm{~g}$ and $0.500 \mathrm{~g} / \mathrm{cm}^{2}$, respectively. The measured area was 45.4 $\pm 0.9 \mathrm{~cm}^{2}$, BMC was $20.1 \pm 0.4 \mathrm{~g}$, and BMD was $0.443 \pm 0.001 \mathrm{~g} / \mathrm{cm}^{2}$, resulting in the recovery of $108 \pm 2 \%, 95 \pm 2 \%$ and $88.5 \pm 0.3 \%$ respectively. An example of the DXA measurement of a gelatin-based bone phantom is displayed in Fig. 2.4, showing a homogeneous distribution of hydroxyapatite within the trabecular bone phantom.

As shown in Fig. 2.5 and Table 2.1, statistically significant association with a strong positive correlation ( $p<0.001$ and $r=0.993)$ was observed between aBMD and strontium content. In 
order to assess the degree of overestimation, the aBMD value of individual measurements were normalized by the mean aBMD value of the reference phantom $\left(0.443 \mathrm{~g} / \mathrm{cm}^{2}\right)$ and subtracted by one. The linear regression between the degree of overestimation and the strontium concentration was fitted through the origin (Fig. 2.6). The degree of overestimation observed from every $1 \%$ strontium substitution of calcium was $13.5 \pm 0.2 \%$ with the $95 \%$ confidence interval of $13.2-13.8$ $\%$. On the contrary, no parameter measured by the clinical or the research QUS system exhibited statistically significant relationship with the strontium concentration (Table 2.1).
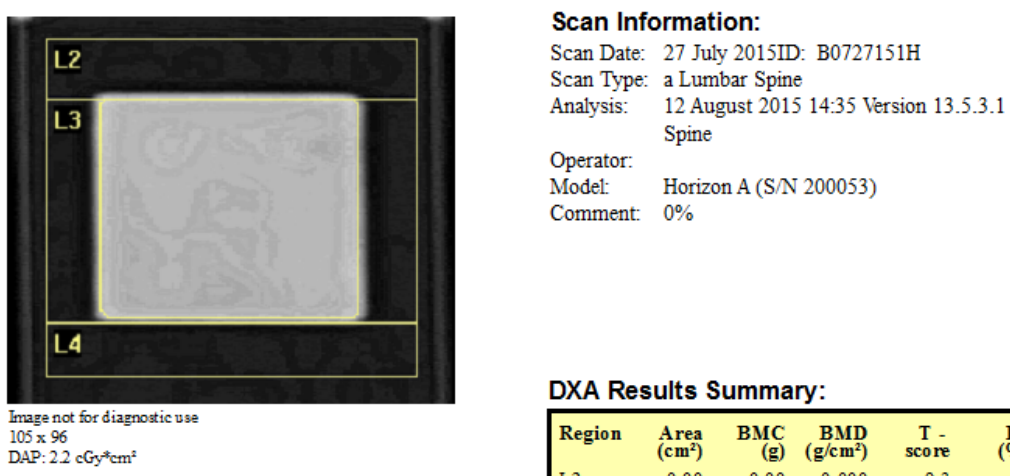

\begin{tabular}{|c|c|c|c|c|c|c|}
\hline Region & $\underset{\left(\mathrm{cm}^{2}\right)}{\text { Area }}$ & $\underset{(\mathrm{g})}{\mathrm{BMC}}$ & $\underset{\left(\mathrm{g} / \mathrm{cm}^{2}\right)}{\mathrm{BMD}}$ & $\underset{\text { score }}{\mathrm{T}}$ & $\begin{array}{c}\text { PR } \\
(\%)\end{array}$ & $\underset{\text { score }}{\mathrm{Z}-}$ \\
\hline L2 & 0.00 & 0.00 & 0.000 & -9.3 & & \\
\hline L3 & 44.92 & 19.89 & 0.443 & -5.8 & 41 & \\
\hline L4 & 0.00 & 0.00 & 0.000 & -10.1 & & \\
\hline Total & 44.92 & 19.89 & 0.443 & -5.8 & 41 & \\
\hline
\end{tabular}

Fig. 2.4. BMD analysis result of the reference phantom by Hologic Horizon ${ }^{\circledR}$ DXA device

Table 2.1. The linear regression analysis for the measurements done on the strontium multilayer trabecular bone phantoms

\begin{tabular}{|c|c|c|c|c|c|c|c|}
\hline & \multirow{2}{*}{$\begin{array}{c}\text { DXA } \\
\text { aBMD }\end{array}$} & \multicolumn{3}{|c|}{ Clinical QUS } & \multicolumn{3}{|c|}{ Research QUS } \\
\hline & & BUA & SOS & SI & BUA & SOS & SI \\
\hline $\begin{array}{l}\text { Regression } \\
\text { Coefficient }\end{array}$ & 0.064 & 0.263 & -0.128 & 0.052 & 0.198 & -0.397 & -0.0814 \\
\hline $\mathrm{p}$ - value & $<0.001^{*}$ & 0.144 & 0.432 & 0.511 & 0.169 & 0.082 & 0.400 \\
\hline$R$ & 0.993 & 0.252 & $0 .-137$ & 0.115 & 0.238 & -0.298 & -0.147 \\
\hline
\end{tabular}

\footnotetext{
* Statistically significant
} 


\begin{tabular}{|c|c|c|}
\hline$\times$ DXA & - Clinical QUS & oResearch QUS \\
\hline
\end{tabular}
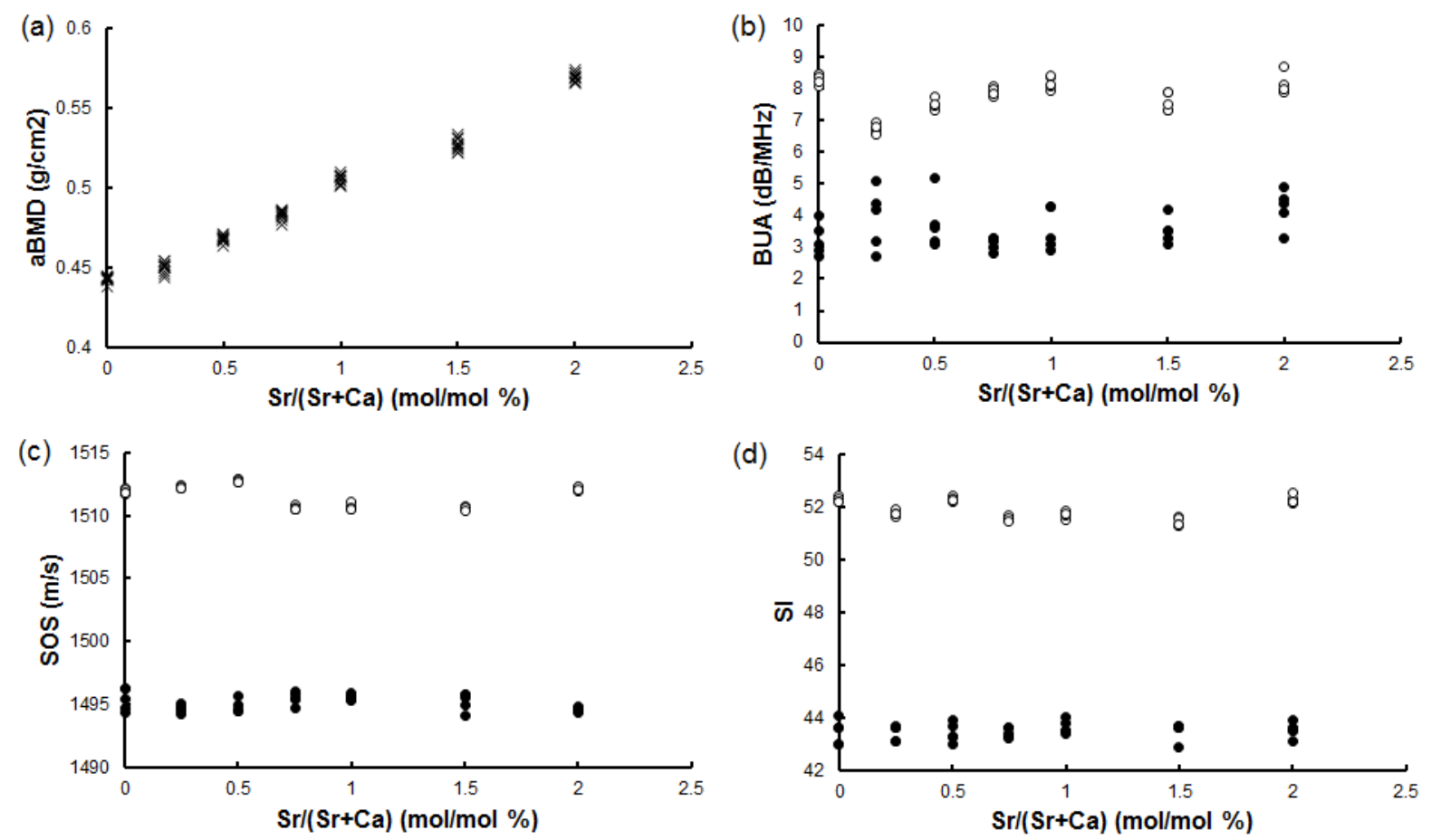

Fig. 2.5. Parameters measured by the DXA system and the two QUS system as a function of strontium concentration: (a) aBMD measured by DXA, (b) BUA measured by QUS, (b) SOS measured by QUS and (d) SI measured by QUS

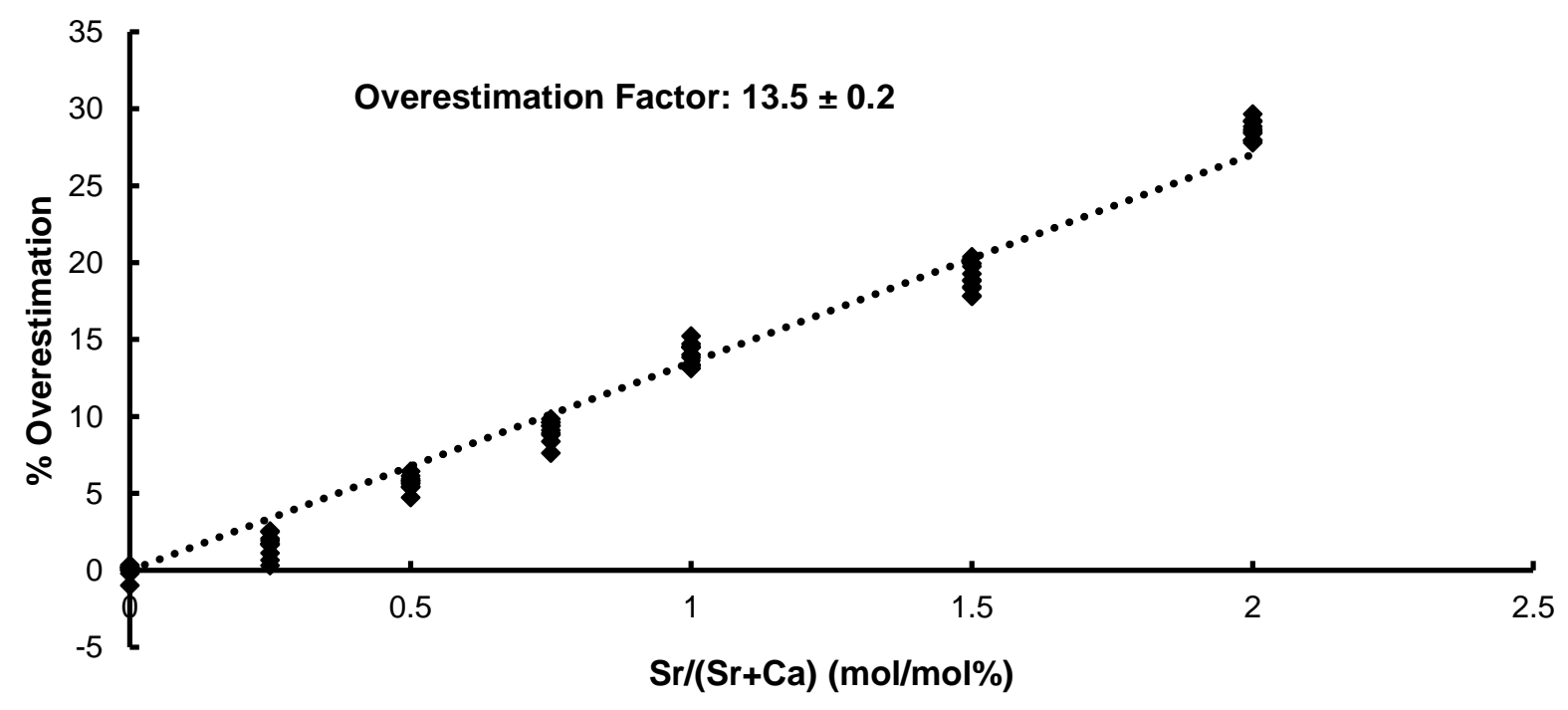

Fig. 2.6. Overestimation of aBMD as a function of strontium content 


\subsubsection{Lead Phantom Measurements}

As shown in Table 2.2 and Fig. 2.7, the relationship between aBMD and lead concentration is shown to be statistically significant weak correlation $(p=0.016, r=-0.347)$. Similarly, statistically significant moderate correlations have been observed in BUA measured by the clinical QUS system $(p<0.001, r=0.658)$ and research QUS system $(p=0.038, r=-0.418)$. However, no statistically significant relationship was observed in SOS and SI measured by the both systems (Table 2.2).

\subsubsection{Aluminum Phantom Measurements}

As shown in Table 2.3 and Fig. 2.8, the effect of aluminum concentration on aBMD measurement is statistically significant $(p<0.001)$ with moderate correlation $(r=-0.549)$. All QUS measurements failed to show statistically significant changes (Table 2.3), except the SOS measured by the clinical system which had a significant moderate correlation $(p=0.006$, $r=-0.530)$.

Table 2.2. The linear regression analysis for the measurements done on the lead multilayer trabecular bone phantoms

\begin{tabular}{|c|c|c|c|c|c|c|c|}
\hline & \multirow{2}{*}{$\begin{array}{c}\text { DXA } \\
\text { aBMD }\end{array}$} & \multicolumn{3}{|c|}{ Clinical QUS } & \multicolumn{3}{|c|}{ Research QUS } \\
\hline & & BUA & SOS & SI & $\overline{B U A}$ & SOS & SI \\
\hline $\begin{array}{l}\text { Regression } \\
\text { Coefficient }\end{array}$ & $-1.7 \times 10^{-5}$ & 0.0072 & -0.0041 & 0.0012 & -0.0043 & 0.0015 & -0.0011 \\
\hline $\mathrm{p}$ - value & $0.016^{*}$ & $<0.001^{*}$ & 0.088 & 0.216 & $0.038^{*}$ & 0.299 & 0.296 \\
\hline$r$ & -0.347 & 0.658 & -0.348 & 0.257 & -0.418 & 0.216 & -0.217 \\
\hline
\end{tabular}

* Statistically significant 


\begin{tabular}{|c|c|c|}
\hline$\times$ DXA & - Clinical QUS & - Research QUS \\
\hline
\end{tabular}
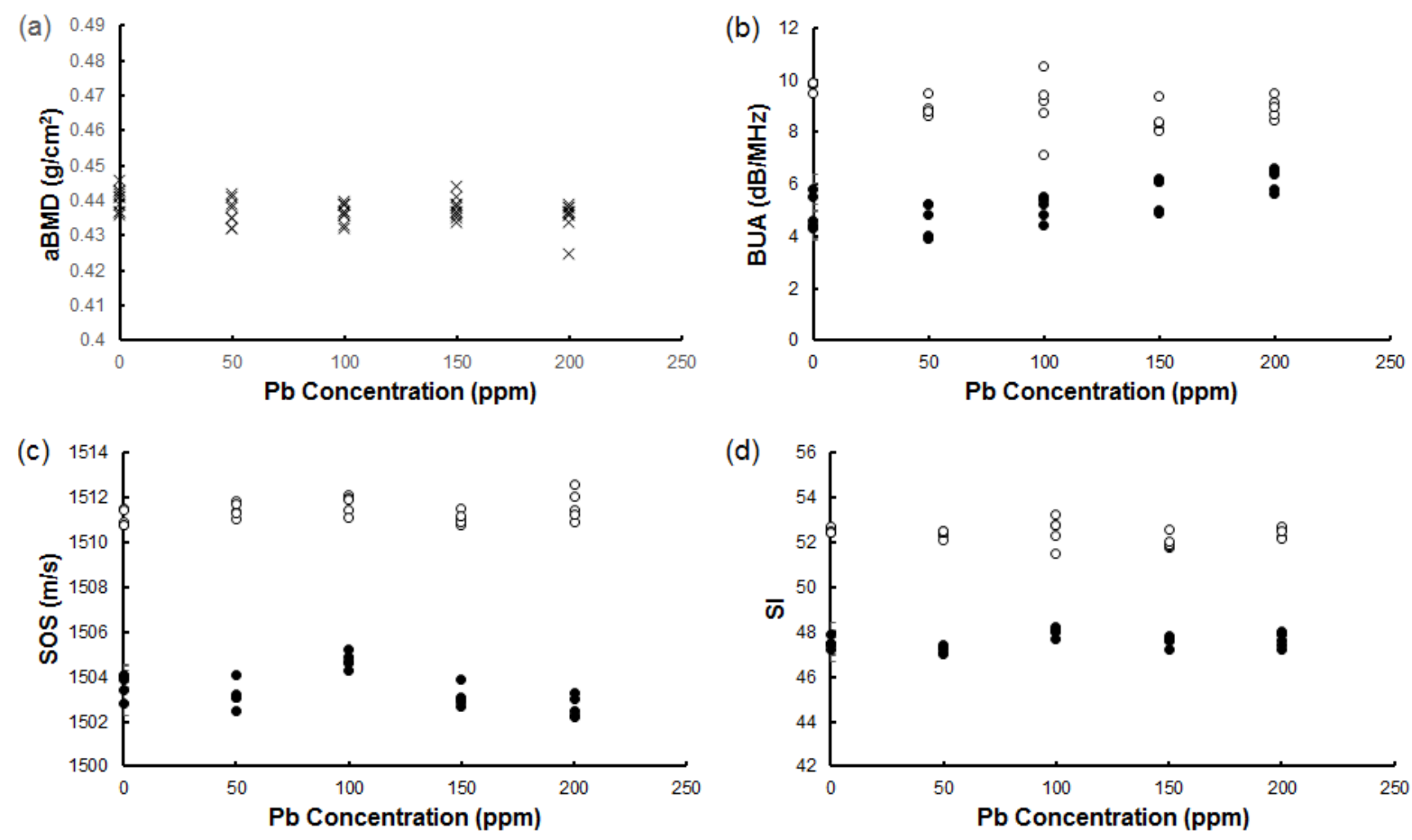

Fig. 2.7. Parameters measured by the DXA system and the two QUS system as a function of lead concentration: (a) aBMD measured by DXA, (b) BUA measured by QUS, (b) SOS measured by QUS and (d) SI measured by QUS

Table 2.3. The linear regression analysis for the measurements done on the aluminum multilayer trabecular bone phantoms

\begin{tabular}{|c|c|c|c|c|c|c|c|}
\hline & \multirow{2}{*}{$\begin{array}{c}\text { DXA } \\
\text { aBMD }\end{array}$} & \multicolumn{3}{|c|}{ Clinical QUS } & \multicolumn{3}{|c|}{ Research QUS } \\
\hline & & BUA & SOS & SI & BUA & SOS & SI \\
\hline $\begin{array}{l}\text { Regression } \\
\text { Coefficient }\end{array}$ & $-3.2 \times 10^{-5}$ & 0.0034 & -0.0047 & -0.0004 & 0.0027 & 0.0042 & 0.0028 \\
\hline $\mathrm{p}$ - value & $<0.001^{*}$ & 0.078 & $0.006^{*}$ & 0.762 & 0.308 & 0.077 & 0.141 \\
\hline$r$ & -0.549 & 0.358 & -0.530 & 0.064 & 0.212 & 0.360 & 0.302 \\
\hline
\end{tabular}

\footnotetext{
${ }^{*}$ Statistically significant
} 

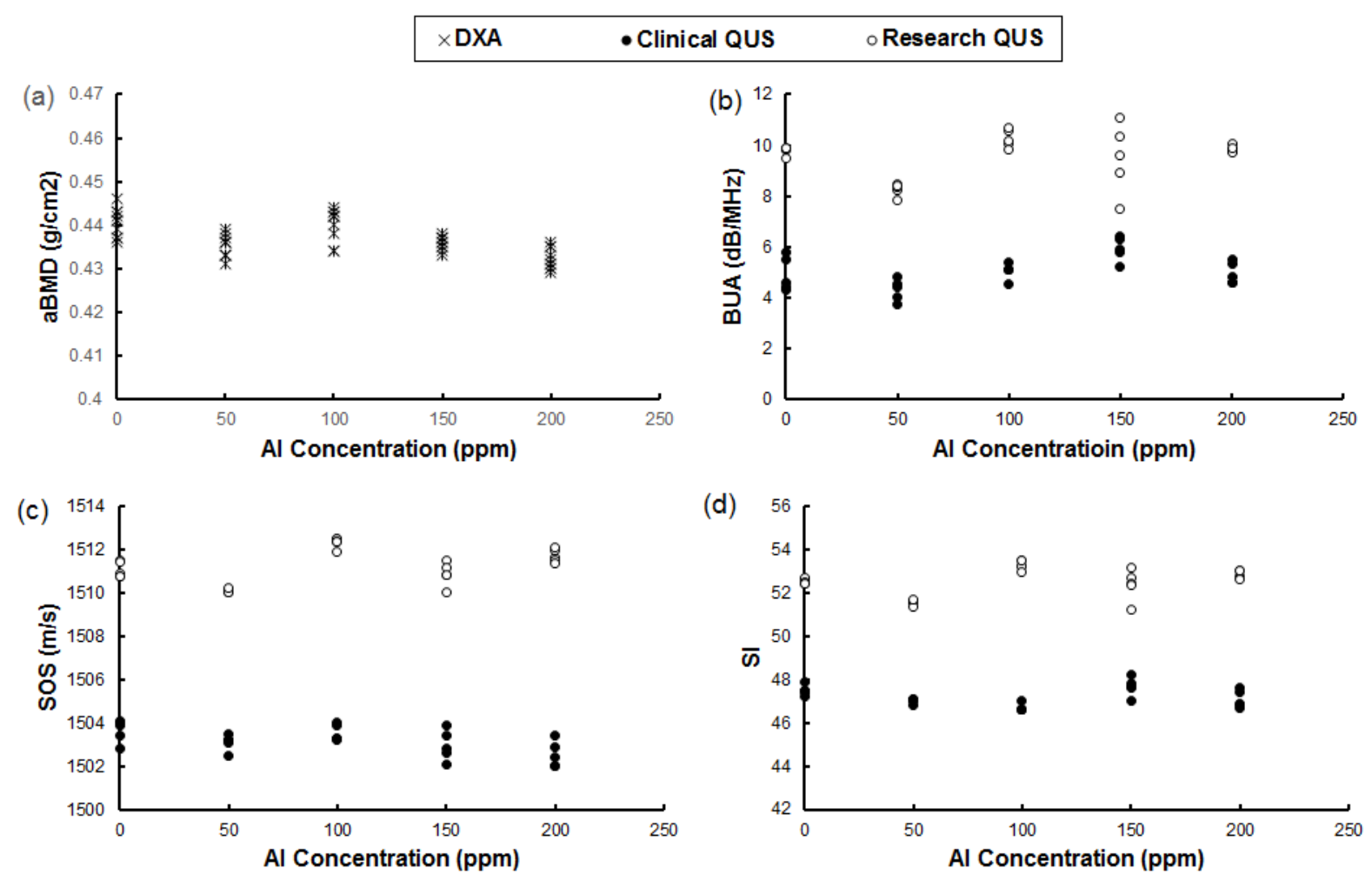

Fig 2.8. Parameters measured by the DXA system and the two QUS system as a function of aluminum concentration: (a) aBMD measured by DXA, (b) BUA measured by QUS, (b) SOS measured by QUS and (d) SI measured by QUS

\subsection{Discussion}

The study demonstrated that the hydroxyapatite-based trabecular bone-mimicking phantom originally designed for QUS is compatible with DXA as well. The images of the phantoms acquired for the BMD analysis indicated homogeneous distribution of the hydroxyapatite within the gelatin medium (Fig. 2.6). BMC and area were estimated with high level of accuracy (95 \pm $2 \%$ and $108 \pm 2 \%$ of recovery, respectively) when the reference phantom without any analyte content was assessed. The overestimation of area might be owing to the divergence of X-ray due 
to the fan beam geometry of Hologic Horizon ${ }^{\circledR}$ device. The observed accuracy of the measurement of BMD was lower than BMC and area $(88.5 \pm 0.3 \%)$. However, the difference in the overestimation factor observed in BMD and BMC was minimal, likely because the strontium content does not tend to influence the estimation of area. ${ }^{(37)}$

In addition, it was verified that the phantoms mimic trabecular bone as the mean BUA and SOS values measured from the reference phantom using the clinical $(3.2 \pm 0.4 \mathrm{~dB} / \mathrm{MHz}$ and 1495.4 $\pm 0.7 \mathrm{~m} / \mathrm{s})$ and research QUS systems $(8.3 \pm 0.2 \mathrm{~dB} / \mathrm{MHz}$ and $1511.0 \pm 0.2 \mathrm{~m} / \mathrm{s})$ were within reported experimental results obtained from human calcaneus. Portero and colleagues ${ }^{(23)}$ assessed excised human calcaneus samples using Hologic Sahara ${ }^{\circledR}$ QUS device and observed BUA values ranging from 0.3 to $80.1 \mathrm{~dB} / \mathrm{MHz}$ and SOS values ranging from 1412 to $1748 \mathrm{~m} / \mathrm{s}$. Although the measured BUA of the trabecular bone-mimicking phantom falls within the range of the reported BUA of human calcaneus, there is a significant difference between the mean value of BUA obtained from the phantom and the human calcaneus. The difference can be attributed to the lack of porous microstructure of bone matrix in the developed homogeneous bone-mimicking phantom, since BUA is not only affected by bone material but also by its microarchitecture. ${ }^{(78,79)}$ However, the phantom can be considered as a good representation of human calcaneus, as a strong positive linear correlation between BUA and vBMD of the trabecular bone-mimicking phantom has been demonstrated by Rizvi and colleagues. ${ }^{(65,66)}$

Upon the assessment of the strontium-substituted phantoms under DXA, a direct linear relationship was obtained between overestimation of aBMD and strontium content; a result consistent with the past studies. ${ }^{(36,37,39)}$ The overestimation factor in our study was determined to be $13.5 \pm 0.3 \%$ per $1 \%$ strontium substitution. Similarly, Liao and colleagues ${ }^{(39)}$ reported the linear overestimation factor of $11.2 \%$ for Hologic Discovery ${ }^{\circledR}$ DXA $(100 / 140 \mathrm{kVp})$. The potential 
source of discrepancy in the overestimation factors between theirs and our studies could be the differences in the phantoms used. In the study by Liao and colleagues, ${ }^{(39)}$ strontium phosphate was added to hydroxyapatite, due to commercial unavailability of strontium hydroxyapatite, to yield the desired molar ratio of $\mathrm{Sr} /(\mathrm{Sr}+\mathrm{Ca})$. In our study, $\mathrm{Ca}(\mathrm{OH})_{2}$ is partially substituted with $\mathrm{Sr}(\mathrm{OH})_{2} \cdot 8 \mathrm{H}_{2} \mathrm{O}$ for the synthesis of hydroxyapatite, and therefore the desired molar ratio of $\mathrm{Sr} /(\mathrm{Sr}+\mathrm{Ca})$ is achieved before the synthesis. Although the exact replication of the overestimation factor was not achieved in this study, it is important to note that the purpose of the strontium phantom measurement with DXA was not to derive the overestimation factor, as it has already been established by several studies. ${ }^{(36,37,39)}$ The primary focus of our study was to verify that the bone phantom is capable of reproducing the aBMD overestimation due to strontium and to allow direct comparison of DXA and QUS measurements obtained from the same phantom.

No statistically significant relationship was found between strontium content and acoustic parameters measured by both the clinical Hologic Sahara ${ }^{\circledR}$ device and the research QUS systems. This suggests that QUS is capable of assessing bone health independently from the clinically relevant level of strontium in bone unlike DXA. Although the strontium overestimation factor of DXA is well established, it has had limited application in clinical practice, since the strontium content is unknown in most clinical cases. Currently, accurate measurement of bone strontium content can only be achieved by bone biopsy, which is a highly invasive technique. Non-invasive techniques such as dual photon absorptiometry (DPA) ${ }^{(80)}$ and X-ray fluorescence $(\mathrm{XRF})^{(32,81)}$ have been suggested, but both techniques have not fully matured to accurately quantify the bone strontium content. Therefore, the use of QUS, which has been demonstrated to be unaffected by the overestimation due to strontium, could potentially be considered in settings where it is 
available, as an adjunct assessment to DXA for monitoring BMD in patients on strontium medications.

Prior to this study, there has been a lack of consensus on the effect of low levels of lead on aBMD measurement using DXA, as there were two distinct studies with conflicting observations; the study by Puzas and colleagues ${ }^{(56)}$ reported $11 \%$ overestimation at 100 ppm, while Popovic and colleagues $^{(43)}$ failed to detect the reported overestimation. The findings of our study is contradicting to the observation made by Puzas and colleagues, ${ }^{(56)}$ but is consistent with the study by Popovic and colleagues. ${ }^{(43)}$ Although the negative relationship is not consistent with the hypothesized overestimation, the deviation of aBMD is within $1 \%$ coefficient of variation (CV) attributed to DXA. Therefore, it can be concluded that the deviation of aBMD is not detectable by DXA for a clinically significant level of lead. As for QUS measurements in our study, BUA was the only parameter that showed moderate correlation with statistical significance. However, it is important to note that the change in the SI values measured by both the clinical and research QUS systems, which determine the estimated aBMD, were statistically insignificant.

Similar to the trend observed for lead, slight decrease in aBMD was observed with respect to the bone aluminum content. Despite the negative correlation, underestimation of aBMD caused by aluminum concentrations up to $100 \mathrm{ppm}$ is under $1 \% \mathrm{CV}$ of the DXA device. Although the aluminum concentrations up to $200 \mathrm{ppm}$ were investigated in this study, the clinically relevant concentration of aluminum in bone typically does not exceed $100 \mathrm{ppm} .{ }^{(82)}$ The exposure of patients with renal-failure to aluminum has been reduced as aluminum-based phosphate binders were replaced with calcium salts in late 1980s. ${ }^{(49)}$ Aluminum was an element of interest because of its hypothesized effect on aBMD underestimation, unlike other bone-seeking elements. In order to further verify this potential underestimation, aluminum phantoms with extreme concentration 
beyond the biologically relevant levels could be assessed. SOS values measured by Hologic Sahara ${ }^{\circledR}$ QUS device showed statistically significant negative correlation, however the SI measured by both the clinical and the research systems remained insensitive to aluminum levels.

\subsection{Conclusions}

This study investigated the effect of strontium, lead and aluminum on DXA and QUS bone density measurements using a trabecular bone-mimicking phantom that is compatible with both modalities. For DXA, linear increase in aBMD with respect to strontium concentration was observed, as expected, with an average overestimation factor of $13.5 \pm 0.2 \%$. In contrast, the two ultrasonic parameters measured by QUS, and SI value derived from the parameters to give estimated aBMD remained independent of strontium content. Therefore, where available, perhaps the use of QUS (BUA and SOS) in addition to DXA assessment, could help provide additional information that aids with monitoring of BMD in patients with a history of or currently on strontium medications. This could potentially aid with identifying an overestimation of patients' aBMD due to strontium content in their bone by DXA. Furthermore, the clinically relevant levels of lead or aluminum were not found to affect either aBMD measured by DXA or SI measured by QUS. The deviation of the parameters due to the clinically relevant levels of lead or aluminum would be indistinguishable from the intrinsic uncertainty associated with the two clinical modalities. Therefore, based on these results, both DXA and QUS can be used to assess the bone health of patients with elevated bone lead or aluminum levels without potential overestimation or underestimation. 


\subsection{Acknowledgements}

Authors would like to thank Arthur Worthington and Bisma Rizvi, both from Dept. of Physics, Ryerson University, for their technical support, and Queenie Wong and Diana Yau from the Centre of Excellence in Skeletal Health Assessment, University of Toronto, for their assistance in the operation of the DXA system. This work is supported by NSERC Discovery grants that were awarded to Dr. Ana Pejović -Milić and Dr. Jahan Tavakkoli. Dr. Angela M. Cheung is supported by a Canada Research Chair (Tier 1) in musculoskeletal and postmenopausal health.

\subsection{Conflict of Interest Disclosure}

The authors have no conflict of interest to report. 


\section{CHAPTER 3:SUMMARY, CONCLUSIONS AND FUTURE WORK}

\subsection{Summary of the study}

When bone-seeking elements such as strontium, lead and aluminum substitute calcium in bone, inaccurate estimation of aBMD by DXA is of potential concern. This phenomenon is well demonstrated by the reported overestimation of aBMD due to strontium ${ }^{(36,37,39)}$, which is taken orally as medication or nutritional supplement for osteoporosis treatment. The deviation in aBMD is attributed to the difference in the mass attenuation coefficients between the incorporated boneseeking elements and calcium, which results in the change in overall attenuation of the two X-ray beams utilized in DXA technique. QUS is another bone densitometry technique, available in bone clinics, that assesses bone mass based on ultrasound parameters, which are not influenced by microscopic changes in the elemental composition of bone. This study aimed to investigate the effect of bone-seeking elements on DXA and QUS measurements using the trabecular bonemimicking phantoms recently developed in our laboratory that contain clinically relevant concentrations of strontium, lead and aluminum.

In this study, the trabecular bone-mimicking phantoms that contain varying concentrations of strontium, lead or aluminum were produced with constant vBMD of $200 \mathrm{mg} / \mathrm{cm}^{3}$, and were assessed using two clinical systems, a Hologic Horizon ${ }^{\circledR}$ DXA device and a Hologic Sahara ${ }^{\circledR}$ QUS device, as well as an in-house research QUS system developed in our laboratory. Upon the assessment of strontium containing bone-mimicking phantoms with DXA, statistically significant linear correlation $(\mathrm{p}<0.001$ and $\mathrm{r}=0.993)$ between $\mathrm{aBMD}$ and the strontium concentration was observed. The aBMD overestimation factor was determined to be $13.5 \pm 0.2 \%$ for every $1 \%$ 
strontium substitution of calcium under DXA with statistical significance $(\mathrm{p}<0.001)$. In contrast, the acoustic parameters BUA, SOS and SI obtained from both QUS devices did not show statistically significant relationship with strontium concentration.

In case of the bone-mimicking phantoms containing lead or aluminum, the aBMD measurement exhibited statistically significant negative relationships with the analyte concentration $(\mathrm{p}=0.016$ and $\mathrm{p}<0.001$ respectively). However, the underestimation aBMD induced by lead concentrations under 200 ppm and aluminum concentrations under 100 ppm were within $1 \% \mathrm{CV}$ intrinsic to a Hologic Horizon ${ }^{\circledR}$ DXA device. Therefore, it could be concluded that the deviation of aBMD due to the clinically relevant levels of lead and aluminum will be indistinguishable for the device. On the other hand, SI obtained from both QUS systems showed no statistically significant correlation with the lead or aluminum concentration in the bonemimicking phantoms. SI, which is based on the linear combination of BUA and SOS (Equation 2.1), holds the clinical significance as it is the parameter derived to yield aBMD comparable to DXA, and subsequently T-score that serves as the basis for the diagnosis of osteoporosis.

Since the QUS measurements are reported to be dependent on not only the bone mass but also the microarchitecture,${ }^{(78,83)}$ one of the limitations of this study is that, the developed bonemimicking phantom lacks the porous trabecular microarchitecture and the cortical bone layer. Therefore, the lack of the porosity in the phantom can contribute to the observed difference between the mean BUA measured from the phantom and the trabecular bone of the calcaneus reported from other published works. ${ }^{(23,24)}$ However, despite of its current limitation, the phantom provides a lot of potential as its main constituent is hydroxyapatite similar to the human bone and therefore behaves similarly with human bone under DXA. In addition, the in-house QUS, a fully researcher controlled system, exhibited the expected results (and in line with the clinical QUS) 
with the addition of strontium, lead or aluminum. Therefore, it supports the physical mechanisms of the ultrasound propagation through the doped bone mimicking phantoms with a constant density.

\subsection{Conclusions}

The study demonstrated that the developed trabecular bone-mimicking phantom is in fact compatible with both DXA and QUS systems which allows a direct comparison of the two modalities. It can be concluded that, unlike DXA measurements, QUS is capable of assessing bone health independently without overestimation of the measured parameters in the presence of strontium. Furthermore, it was determined that the clinically relevant concentrations of lead and aluminum do not induce statistically significant deviation in the parameter aBMD measured by DXA and SI measured by QUS techniques. In case of aBMD, the observed statistically significant deviations from clinically relevant concentrations of lead and aluminum cannot be detected under DXA because the deviations are within the $1 \% \mathrm{CV}$ of DXA.

\subsection{Future Work}

Although this study was successful in proving the hypothesis, it also opened up new questions and opportunity for the future work. The first could be the verification of hypothesized effect of lead and aluminum on aBMD, which are overestimation and underestimation, respectively. This work will aim to show that the two elements can in fact cause the deviation of aBMD at higher concentration, and can be achieved by producing phantoms containing high concentration of lead and aluminum beyond the clinically relevant range present in patients. 
Furthermore, the first generation of the developed trabecular bone-mimicking phantom can be improved to replicate the human calcaneus more accurately. As mentioned the limitation of the current phantom model is the lack of the porous microarchitecture. The simulation of porosity might be achieved by dispersing beads of material that mimics the ultrasonic property of bone marrow within the gelatin and hydroxyapatite mixture before solidification. Clarke and colleagues demonstrated a similar approach where granules of gelatin that simulated the marrow was dispersed within epoxy medium which simulated the bone. ${ }^{(57)}$ Additional improvement of the bone phantoms will be to include the cortical layer present at the bone site used for the measurement.

Another future step of this project should consider the axial transmission QUS technique. ${ }^{(84)}$ The axial transmission QUS system is a more recently developed technique which assess SOS of the shear wave generated and propagated in the cortical layer of the bones such as the phalanx, the radius, and the tibia. The addition of the cortical layer to our current bone-mimicking phantom would be necessary to make the phantom compatible with this QUS technique. Development of the phantom compatible with the axial transmission QUS system would allow thorough investigation of this newly developed technology and its comparison with the DXA and the standard QUS techniques. 


\section{APPENDIX A: Procedure for Producing Trabecular Bone- mimicking Phantoms}

1. $1 \mathrm{M} \mathrm{HPO}_{4}{ }^{2-}$ setting solution was produced by dissolving $\mathrm{Na}_{2} \mathrm{HPO}_{4}$ into MiliQ water

2. The strontium substituted hydroxyapatite bone phantoms were produced by mixing $\mathrm{CaHPO}_{4} \cdot 2 \mathrm{H}_{2} \mathrm{O}, \mathrm{Ca}(\mathrm{OH})_{2}$ and $\mathrm{Sr}(\mathrm{OH})_{2} \cdot 8 \mathrm{H}_{2} \mathrm{O}$, while keeping constant $(\mathrm{Ca}+\mathrm{Sr}) / \mathrm{P}$ molar ratio of 1.67. The $1 \mathrm{M}$ setting solution was added to the mixture of the powders to achieve powder-toliquid ratio of 2:1.

3. The hydroxyapatite bone phantoms doped with lead or aluminum were synthesized by mixing $\mathrm{CaHPO}_{4} \cdot 2 \mathrm{H}_{2} \mathrm{O}$ and $\mathrm{Ca}(\mathrm{OH})_{2}$ with constant $\mathrm{Ca} / \mathrm{P}$ molar ratio of 1.67 and adding $1000 \mu \mathrm{g} / \mathrm{mL}$ lead or aluminum standard solution to the mixture to achieve desired concentration of the analytes. The $1 \mathrm{M}$ setting solution was added to the mixture of the powders to achieve a powder-to-liquid ratio of 2:1.

4. The hydroxyapatite phantoms were set to cure for two weeks. Once set, the hydroxyapatite phantoms were dried in an oven at $80^{\circ} \mathrm{C}$ until there is no apparent change in weight due to the loss of water.

5. The dried phantoms were powdered in a ball mill using a rotating rate of 30 cycles/second.

6. The powdered phantoms were added to $5 \% \mathrm{w} / \mathrm{w}$ porcine gelatin solution and poured into an airtight container with dimensions of $6.5 \times 6.5 \times 2.5 \mathrm{~cm}^{3}$

7. The mixtures were rotated for two hours to ensure that a homogeneous distribution of the hydroxyapatite particles within the gelatin medium was achieved and to prevent aggregation and sedimentation of the particles.

8. The phantoms were cooled down in a refrigerator at $4^{\circ} \mathrm{C}$ for 12 hours. Once the phantoms were solidified, they were removed from the container. 


\section{REFERENCES}

1. Jin T, Nordberg G, Ye T, Bo M, Wang H, Zhu G, et al. Osteoporosis and renal dysfunction in a general population exposed to cadmium in China. Environ Res. 2004;96(3):353-9.

2. Kazantzis G. Cadmium, osteoporosis and calcium metabolism. BioMetals. 2004;17(5):493-8.

3. Adachi JD, Ioannidis G, Pickard L, Berger C, Prior JC, Joseph L, et al. The association between osteoporotic fractures and health-related quality of life as measured by the Health Utilities Index in the Canadian Multicentre Osteoporosis Study (CaMos). Osteoporos Int. 2003;14(11):895-904.

4. Korpi-Steiner N, Milhorn D, Hammett-Stabler C. Osteoporosis in men. Clin Biochem. 2014;47:950-9.

5. Brown JP, Josse RG. 2002 clinical practice guidelines for the diagnosis and management of osteoporosis in Canada. CMAJ. 2002;167(10 suppl):s1-34.

6. Wiktorowicz ME, Goeree R, Papaioannou A, Adachi JD, Papadimitropoulos E. Economic implications of hip fracture: health service use, institutional care and cost in Canada. Osteoporos Int. 2001;12(4):271-8.

7. Tarride JE, Hopkins RB, Leslie WD, Morin S, Adachi JD, Papaioannou A, et al. The burden of illness of osteoporosis in Canada. Osteoporos Int. 2012;23(11):2591-600.

8. Blake GM, Fogelman I. An update on dual-energy x-ray absorptiometry. Semin Nucl Med. Elsevier Inc.; 2010 Jan;40(1):62-73.

9. Blake GM, Fogelman I. Technical principles of dual energy x-ray absorptiometry. Semin Nucl Med. 1997;27(3):210-28.

10. Podgorsak EB. Radiation Physics for Medical Physcists. 2010.

11. Kalender W, Engelke K, Fuerst TP, Glüer C-C, Laugier P, Shepherd J. 5. X-ray Absorptiometry. J ICRU. 2009;9(1):37-58.

12. Ozdemir A, Uçar M. Standardization of spine and hip BMD measurements in different DXA devices. Eur J Radiol. 2007;62(3):423-6.

13. Lu Y, Fuerst T, Hui S, Genant HK. International Original Article Standardization of Bone Mineral Density at Femoral Neck, Trochanter and Ward's Triangle. Osteoporos Int. 2001;12:438-44.

14. Fan B, Lu Y, Genant H, Fuerst T, Shepherd J. Does standardized BMD still remove differences between Hologic and GE-Lunar state-of-the-art DXA systems? Osteoporos Int. 2010;21(7):1227-36. 
15. Laugier P, Haïatt G. Bone quantitative ultrasound. 1st ed. Laugier P, Haïat G, editors. Bone Quantitative Ultrasound. Springer Netherlands; 2011. 1-468 p.

16. Lentle B, Cheung AM, Hanley DA, Leslie WD, Lyons D, Papaioannou A, et al. Osteoporosis Canada 2010 guidelines for the assessment of fracture risk. Can Assoc Radiol J. Elsevier Inc.; 2011;62(4):243-50.

17. Kanis JA, McCloskey E V., Johansson H, Oden A, Melton LJ, Khaltaev N. A reference standard for the description of osteoporosis. Bone. 2008;42(3):467-75.

18. Nayak S, Olkin I, Liu H, Grabe M, Gould MK, Allen IE, et al. Meta-analysis: Accuracy of quantitative ultrasound for identifying patients with osteoporosis. Ann Intern Med. 2006;144(11):832-41.

19. Kalender W, Engelke K, Fuerst TP, Glüer C-C, Laugier P, Shepherd J. 7. Quantitative Ultrasound. J ICRU. 2009;9(1):71-104.

20. Chen P-J, Chen T, Lu M-C, Yao W-J. The measurements of ultrasound parameters on calcaneus by two-sided interrogation techniques. Meas Sci Technol. 2005;16(6):1349-54.

21. Langton CM, Njeh CF. The measurement of broadband ultrasonic attenuation in cancellous bone--a review of the science and technology. IEEE Trans Ultrason Ferroelectr Freq Control. 2008;55(7):1546-54.

22. Laugier P, Droin P, Laval-Jeantet a M, Berger G. In vitro assessment of the relationship between acoustic properties and bone mass density of the calcaneus by comparison of ultrasound parametric imaging and quantitative computed tomography. Bone. 1997;20(2):157-65.

23. Portero-Muzy NR, Chavassieux PM, Mitton D, Duboeuf F, Delmas PD, Meunier PJ. Eulerstrut.cavity, a new histomorphometric parameter of connectivity reflects bone strength and speed of sound in trabecular bone from human os calcis. Calcif Tissue Int. 2007;81(2):92-8.

24. Bouxsein ML, Radloff SE. Quantitative ultrasound of the calcaneus reflects the mechanical properties of calcaneal trabecular bone. J Bone Miner Res. 1997;12(5):839-46.

25. Njeh CF, Boivin CM, Langton CM. The role of ultrasound in the assessment of osteoporosis: A review. Osteoporos Int. 1997;7(1):7-22.

26. Kovač D, Lindič J, Kandus A, Bren FA. Quantitative ultrasound of the calcaneus and dual X-ray absorptiometry of the lumbar spine in assessment and follow-up of skeletal status in patients after kidney transplantation. Osteoporos Int. 2003;14(2):166-70.

27. Hadji P, Hars O, Wüster C, Bock K, Alberts US, Bohnet HG, et al. Stiffness index identifies patients with 
osteoporotic fractures better than ultrasound velocity or attenuation alone. Maturitas. 1999;31(3):221-6.

28. Xu Y, Guo B, Gong J, Xu H, Bai Z. The correlation between calcaneus stiffness index calculated by QUS and total body BMD assessed by DXA in Chinese children and adolescents. J Bone Miner Metab. Springer-Verlag Tokyo; 2014 Mar;32(2):159-66.

29. Hologic Inc. SAHARA Clinical Bone Sonometer: User's Guide. 1998.

30. O'Flaherty EJ. Physiologically Based Models for Bone-Seeking. Toxicol Appl Pharmacol. 1991;111(2):299_ 312.

31. Meunier PJ, Roux C, Seeman E, Ortolani S, Badurski JE, Spector TD, et al. The effects of strontium ranelate on the risk of vertebral fracture in women with postmenopausal osteoporosis. N Engl J Med. 2004;350(5):45968.

32. Moise H, Chettle DR, Pejović-Milić A. Monitoring bone strontium intake in osteoporotic females selfsupplementing with strontium citrate with a novel in-vivo X-ray fluorescence based diagnostic tool. Bone. 2014;61:48-54.

33. Fonseca JE. Rebalancing bone turnover in favour of formation with strontium ranelate: Implications for bone strength. Rheumatology. 2008;47(SUPPL. 4):19-21.

34. Reginster JY, Seeman E, De Vernejoul MC, Adami S, Compston J, Phenekos C, et al. Strontium ranelate reduces the risk of nonvertebral fractures in postmenopausal women with osteoporosis: Treatment of Peripheral Osteoporosis (TROPOS) study. J Clin Endocrinol Metab. 2005;90(5):2816-22.

35. Boivin G, Farlay D, Khebbab MT, Jaurand X, Delmas PD, Meunier PJ. In osteoporotic women treated with strontium ranelate, strontium is located in bone formed during treatment with a maintained degree of mineralization. Osteoporos Int. 2010;21(4):667-77.

36. Christoffersen J, Christoffersen MR, Kolthoff N, Bärenholdt O. Effects of strontium ions on growth and dissolution of hydroxyapatite and on bone mineral detection. Bone. 1997;20(1):47-54.

37. Nielsen SP, Slosman D, Helmer O, Meunier PJ. Influence of Strontium on Bone Mineral Density and Bone Mineral Content Measurements by Dual X-Ray Absorptiometry. J Clin Densitom. 1999;2(4):371-9.

38. Blake GM, Fogelman I. The Correction of BMD Measurements for Bone Strontium Content. J Clin Densitom. 2007;10(3):259-65. 
39. Liao J, Blake GM, McGregor AH, Patel R. The effect of bone strontium on BMD is different for different manufacturers’ DXA Systems. Bone. Elsevier Inc.; 2010 Nov;47(5):882-7.

40. Berglund M, Åkesson A, Bjellerup P, Vahter M. Metal-bone interactions. Toxicol Lett. 2000;112-113:21925.

41. Sun Y, Sun D, Zhou Z, Zhu G, Zhang H, Chang X, et al. Osteoporosis in a Chinese population due to occupational exposure to lead. Am J Ind Med. 2008 Jun;51(6):436-42.

42. Potula V, Kleinbaum D, Kaye W. Lead exposure and spine bone mineral density. J Occup Environ Med. 2006 Jun;48(6):556-64.

43. Popovic M, McNeill FE, Webber CE, Chettle DR. The effect of lead in bone densitometry. Nucl Instruments Methods Phys Res Sect B Beam Interact with Mater Atoms. 2004;213:599-602.

44. Popovic M, McNeill FE, Chettle DR, Webber CE, Lee CV, Kaye WE. Impact of Occupational Exposure on Lead Levels in Women. Environ Health Perspect. 2005 Feb 9;113(4):478-84.

45. Baranowska I, Czernicki K, Aleksandrowicz R. The analysis of lead, cadmium, zinc, copper and nickel content in human bones from the Upper Silesian industrial district. Sci Total Environ. 1995;159(2-3):155-62.

46. Pichery C, Bellanger M, Zmirou-Navier D, Glorennec P, Hartemann P, Grandjean P. Childhood lead exposure in France: benefit estimation and partial cost-benefit analysis of lead hazard control. Environ Health. 2011;10:44.

47. Mohseni HK, Cowan D, Chettle DR, Pejović-Milić A, Priest N, Matysiak W, et al. A Pilot Study Measuring Aluminum in Bone in Alzheimer's Disease and control Subjects Using in vivo Neutron Activation Analysis. 2016;53:933-42.

48. Exley C, Vickers T. Elevated brain aluminium and early onset Alzheimer's disease in an individual occupationally exposed to aluminium: a case report. J Med Case Rep. Journal of Medical Case Reports; 2014;8(1):41.

49. Malluche HH. Aluminium and bone disease in chronic renal failure. Nephrol Dial Transplant. 2002;17 Suppl 2:21-4.

50. Pei Y, Hercz G, Greenwood C, Sherrard D, Segre G, Manuel A, et al. Non-invasive prediction of aluminum bone disease in hemo- and peritoneal dialysis patients. Kidney Int. 1992;41(5):1374-82. 
51. Salusky IB, Coburn JW, Brill J, Foley J, Slatopolsky E, Fine RN, et al. Bone disease in pediatric patients undergoing dialysis with CAPD or CCPD. Kidney Int. 1988;33:975-82.

52. Cannata-Andía JB, Fernández-Martín JL. The clinical impact of aluminium overload in renal failure. Nephrol Dial Transplant. 2002;17 Suppl 2:9-12.

53. Hellström HO, Mjöberg B, Mallmin H, Michaëlsson K. The aluminum content of bone increases with age, but is not higher in hip fracture cases with and without dementia compared to controls. Osteoporos Int. 2005;16(12):1982-8.

54. Bondy SC. The neurotoxicity of environmental aluminum is still an issue. Neurotoxicology. Elsevier B.V.; 2010;31(5):575-81.

55. Cournot-Witmer G, Zingraff J, Plachot JJ, Escaig F, Lefèvre R, Boumati P, et al. Aluminum localization in bone from hemodialyzed patients: relationship to matrix mineralization. Kidney Int. 1981;20(3):375-8.

56. Puzas JE, Campbell JR, O’Keefe RJ, Schwarz EM, Zuscik MJ, Rosier RN. Lead in the skeleton interferes with the accurate measurement of bone mineral density by DEXA [Abstract]. Journal of Bone and Mineral Metabolism. 2002.

57. Clarke AJ, Evans JA, Truscott JG, Milner R, Smith MA. A phantom for quantitative ultrasound of trabecular bone. Phys Med Biol. 1994;39(10):1677-87.

58. Hodgskinson R, Njeh CF, Whitehead $\mathrm{M}$ a, Langton CM. The non-linear relationship between BUA and porosity in cancellous bone. Phys Med Biol. 1996;41(11):2411-20.

59. Tatarinov a., Pontaga I, Vilks U. Modeling the influence of mineral content and porosity on ultrasound parameters in bone by using synthetic phantoms. Mech Compos Mater. 1999;35(2):147-54.

60. Zhang C, Le LH, Zheng R, Ta D, Lou E. Measurements of ultrasonic phase velocities and attenuation of slow waves in cellular aluminum foams as cancellous bone-mimicking phantoms. $\mathrm{J}$ Acoust Soc Am. 2011;129(5):3317-26.

61. Wear $\mathrm{K}$ a. The dependencies of phase velocity and dispersion on trabecular thickness and spacing in trabecular bone-mimicking phantoms. J Acoust Soc Am. 2005;118(2):1186-92.

62. Lee K Il, Choi MJ. Phase velocity and normalized broadband ultrasonic attenuation in Polyacetal cuboid bonemimicking phantoms. J Acoust Soc Am. 2007;121(6):EL263-9. 
63. Da Silva E, Kirkham B, Heyd D V., Pejović-Milić A. Pure hydroxyapatite phantoms for the calibration of in vivo X-ray fluorescence systems of bone lead and strontium quantification. Anal Chem. 2013;85(19):918995.

64. Da Silva E, Heyd D V, Rizvi B, Pejović-Milić A. The preparation of strontium-substituted hydroxyapatite bone phantoms with high strontium concentrations. Biomed Phys Eng Express. 2016;2(1):015006.

65. Rizvi B, Da Silva E, Tavakkoli J, Pejović-Milić A, Slatkovska L, Cheung AM. Bone mineral density measurements of strontium-rich trabecular bone-mimicking phantoms using quantitative ultrasound. Med Phys. 2016 (submitted and under review)

66. Rizvi B. Investigating The Effect Of Strontium In Bone Mineral Density Measurements Of Trabecular Bone Mimicking Phantoms Using Quantitative Ultrasound. MSc Thesis. Ryerson University; 2016.

67. E. H. Jeffery, K. Abreo EB. Systemic Aluminum Toxicity: Effects on Bone, Hematopoietic Tissue, and Kidney. J Toxicol Environ Health. 1996;48(6):649-66.

68. Hans D, Fuerst T, Duboeuf F. Quantitative ultrasound bone measurement. Eur Radiol. 1997;7(10):43-50.

69. Prins SH, Jørgensen HL, Jørgensen L V, Hassager C. The role of quantitative ultrasound in the assessment of bone: a review. Clin Physiol. 1998;18(1):3-17.

70. Dane C, Dane B, Cetin A, Erginbas M. The role of quantitative ultrasound in predicting osteoporosis defined by dual-energy X-ray absorptiometry in pre- and postmenopausal women. Climacteric. 2008;11(4):296-303.

71. Trimpou P, Bosaeus I, Bengtsson BA, Landin-Wilhelmsen K. High correlation between quantitative ultrasound and DXA during 7 years of follow-up. Eur J Radiol. 2010;73(2):360-4.

72. Glüer CC, Eastell R, Reid DM, Felsenberg D, Roux C, Barkmann R, et al. Association of five quantitative ultrasound devices and bone densitometry with osteoporotic vertebral fractures in a population-based sample: the OPUS Study. J Bone Miner Res. 2004;19(5):782-93.

73. Edelmann-Schäfer B, Berthold LD, Stracke H, Lührmann PM, Neuhäuser-Berthold M. Identifying elderly women with osteoporosis by spinal dual x-ray absorptiometry, calcaneal quantitative ultrasound and spinal quantitative computed tomography: A comparative study. Ultrasound Med Biol. 2011;37(1):29-36.

74. Dahl SG, Allain P, Marie PJ, Mauras Y, Boivin G, Ammann P, et al. Incorporation and distribution of strontium in bone. Bone. 2001;28(4):446-53. 
75. Bärenholdt O, Kolthoff N, Nielsen SP. Effect of long-term treatment with strontium ranelate on bone strontium content. Bone. Elsevier Inc.; 2009 Aug;45(2):200-6.

76. Chappard C, Camus E, Lefebvre F, Guillot G, Bittoun J, Berger G, et al. Evaluation of error bounds on calcaneal speed of sound caused by surrounding soft tissue. J Clin Densitom. 2000;3(2):121-31.

77. Pejović-Milić A, Brito JA, Gyorffy J, Chettle DR. Ultrasound measurements of overlying soft tissue thickness at four skeletal sites suitable for in vivo x-ray fluorescence. Med Phys. 2002;29(11):2687.

78. Cortet B, Boutry N, Dubois P, Legroux-Gerot I, Corten A, Marchandise X. Does Quantitative Ultrasound of Bone Reflect More Bone Mineral Density Than Bone Microarchitecture? Calcif Tissue Int. 2004;74(1):60-7.

79. Sasso M, Haïatt G, Yamato Y, Naili S, Matsukawa M. Dependence of ultrasonic attenuation on bone mass and microstructure in bovine cortical bone. J Biomech. 2008;41(2):347-55.

80. Nielsen SP, Bärenholdt O, Bärenholdt-Schiøler C, Mauras Y, Allain P. Noninvasive Measurement of Bone Strontium. J Clin Densitom. 2004;7(3):262-8.

81. Moise H, Adachi JD, Chettle DR, Pejović-Milić A. Monitoring bone strontium levels of an osteoporotic subject due to self-administration of strontium citrate with a novel diagnostic tool, in vivo XRF: A case study. Bone. 2012;51(1):93-7.

82. D’Haese PC, Couttenye MM, Lamberts L V., Elseviers MM, Goodman WG, Schrooten I, et al. Aluminum, iron, lead, cadmium, copper, zinc, chromium, magnesium, strontium, and calcium content in bone of endstage renal failure patients. Clin Chem. 1999;45(9):1548-56.

83. Hans D, Arlot ME, Schott AM, Roux JP, Kotzki PO, Meunier PJ. Do ultrasound measurements on the os calcis reflect more the bone microarchitecture than the bone mass?: A two-dimensional histomorphometric study. Bone. 1995;16(3):295-300.

84. Barkmann R, Kantorovich E, Singal C, Hans D, Genant HK, Heller M, et al. A new method for quantitative ultrasound measurements at multiple skeletal sites: first results of precision and fracture discrimination. J Clin Densitom. 2000;3(1):1-7. 